TRANSACTIONS OF THE

AMERICAN MATHEMATICAL SOCIETY

Volume 355, Number 4, Pages 1593-1620

S 0002-9947(02)03188-4

Article electronically published on November 14, 2002

\title{
MONOMIAL BASES FOR $q$-SCHUR ALGEBRAS
}

\author{
JIE DU AND BRIAN PARSHALL
}

\begin{abstract}
Using the Beilinson-Lusztig-MacPherson construction of the quantized enveloping algebra of $\mathfrak{g l}_{n}$ and its associated monomial basis, we investigate $q$-Schur algebras $\mathbf{S}_{q}(n, r)$ as "little quantum groups". We give a presentation for $\mathbf{S}_{q}(n, r)$ and obtain a new basis for the integral $q$-Schur algebra $S_{q}(n, r)$, which consists of certain monomials in the original generators. Finally, when $n \geqslant r$, we interpret the Hecke algebra part of the monomial basis for $S_{q}(n, r)$ in terms of Kazhdan-Lusztig basis elements.
\end{abstract}

\section{INTRODUCTION}

Let $\mathbf{U}=\mathbf{U}(\mathfrak{g})$ be the quantized enveloping algebra over $\mathbb{Q}(v)$ associated to a finite-dimensional complex semisimple Lie algebra $\mathfrak{g}$, and let $U$ be its Lusztig $\mathcal{Z}$ form, where $\mathcal{Z}=\mathbb{Z}\left[v, v^{-1}\right]$. When $\mathfrak{g}$ has a simply laced root system, monomial bases for the positive (resp., negative) part $U^{+}$(resp., $U^{-}$) of $U$ have been constructed in [19, 7.8] using the theory of Ringel-Hall algebras; see [3] for an approach that works for all finite types. For example, the monomial basis for $U^{+}$consists of certain explicit (ordered) monomials in the standard generators for $U^{+}$. The algebra $\mathbf{U}$ also has a PBW-type basis, but monomial bases are simpler than PBW bases and, in addition, they are closely related to canonical (or crystal) bases.

Let $\mathbf{S}_{q}(n, r)$ be a $q$-Schur algebra over $\mathbb{Q}(v)$; see below for the definition of $\mathbf{S}_{q}(n, r)$ and the associated Hecke algebra $\mathbf{H}=\mathbf{H}\left(\mathfrak{S}_{r}\right)$. The $q$-Schur algebras were introduced by Dipper and James [5], [6] (see [16] for an earlier version in the context of quantum groups). These algebras, as well as their analogues over other fields, play an important role in the non-defining representation and cohomology theories of the finite general linear groups. It is natural to ask how to construct monomial bases for the $\mathbf{S}_{q}(n, r)$. Using a beautiful geometric setting for $q$-Schur algebras, Beilinson, Lusztig, and MacPherson [1] studied the quantized enveloping algebra $\mathbf{U}=\mathbf{U}\left(\mathfrak{g l}_{n}\right)$ of the reductive Lie algebra $\mathfrak{g l}_{n}$ as a "limit" of $q$-Schur algebras and described a monomial basis for $\mathbf{U}$ in terms of another basis whose elements are formal infinite sums indexed by certain $n \times n$ matrices over $\mathbb{Z}$. In particular, there is a natural surjection $\mathbf{U} \rightarrow \mathbf{S}_{q}(n, r)$, arising as a "truncation" map; it carries an infinite sum in $\mathbf{U}$ to a finite sum in $\mathbf{S}_{q}(n, r)$. In addition, the results of [1] have an integral version (i.e., over $\mathcal{Z}$ ) 9 , and there is a corresponding surjection $U \rightarrow S_{q}(n, r)$. Eventually, this work leads to a quantum Weyl reciprocity [11, also valid at the integral level.

Received by the editors October 1, 2001 and, in revised form, July 1, 2002.

2000 Mathematics Subject Classification. Primary 17B37, 20C08, 20 G05.

Supported partially by ARC and NSF. 
This paper applies the approach of [1] to obtain a natural monomial basis for $\mathbf{S}_{q}(n, r)$. Thus, Theorems 5.4 and 6.4 present monomial bases for $\mathbf{S}_{q}(n, r)$ and its integral version $S_{q}(n, r)$, while Theorem 5.5 describes the monomial basis elements in terms of certain elements constructed in [1]. As we show elsewhere in the paper, these bases are very natural and enjoy nice properties not shared by the PBW basis; see, e.g., 4.97 .29 .4 For example, if $n \geqslant r$, then $\mathbf{H} \subset \mathbf{S}_{q}(n, r)$, and Theorem 9.4 shows how the monomial basis "restricts" to a monomial basis for $\mathbf{H}$ (given as monomials in the Kazhdan-Lusztig elements $C_{s}^{\prime} \in \mathbf{H}[15]$ ).

This work was initially motivated by [7] (as well as by an announcement of the results in 8 by Doty at the 2001 New Orleans AMS meeting). Their work gives an explicit presentation of $\mathbf{S}_{q}(n, r)$ as well as a PBW-type basis $\mathbf{S}_{q}(n, r)$. We were motivated to see how to cast these results in the more geometric setting of [1]. Both a presentation (in a slightly different form) and a PBW basis also can be obtained as a new application of [1]; see Theorems 5.4 and 6.6.

The table below displays three different bases for $S_{q}(n, r)$, indicating how they stand in relation to bases for both the integral quantum enveloping algebra $U_{\mathcal{Z}}\left(\mathfrak{g l}_{n}\right)$ and the integral Hecke algebra $H\left(\mathfrak{S}_{r}\right)$ with $r \leqslant n$.

\begin{tabular}{|c|c|l|}
\hline$H\left(\mathfrak{S}_{r}\right)$ & $S_{q}(n, r)$ & \multicolumn{1}{|c|}{$U_{\mathcal{Z}}\left(\mathfrak{g l}_{n}\right)$} \\
\hline \hline$\tilde{T}_{w}$ & {$[A]$} & PBW basis \\
\hline$C_{w}^{\prime}$ & $\{A\}$ & Canonical basis $\left(\right.$ for $\left.U_{\mathcal{Z}}^{+}\left(\mathfrak{g l}_{n}\right)\right)$ \\
\hline$C_{s_{1}}^{\prime} \cdots C_{s_{k}}^{\prime}$ & $\mathrm{m}^{(A)}$ & Monomial basis \\
\hline
\end{tabular}

The "orbital" basis elements $[A]$ are indexed by $n \times n$ matrices over $\mathbb{N}$ whose entries sum to $r$. This basis is the normalized version of the usual standard basis for a centralizer algebra, whose elements are denoted by $\phi_{\lambda \mu}^{d}$ in [6] 1.4]. Hence, its Hecke algebra counterpart consists of the normalized basis elements $\tilde{T}_{w}=v^{-\ell(w)} T_{w}$. In $U_{\mathcal{Z}}\left(\mathfrak{g l}_{n}\right)$, this basis corresponds to a PBW basis by means of its connection with a canonical (or crystal) basis; see [14] for further connections. All three algebras have canonical bases indicated in the second row in the table. They arise naturally from the corresponding monomial bases.

This work provides a foundation for [12], which directly relates the geometric approach [1] to the theory of Ringel-Hall algebras for linear quivers. In particular, this leads to a new connection between the theories of Ringel-Hall algebras and $q$-Schur algebras.

Some notation. Throughout, $\mathcal{Z}=\mathbb{Z}\left[v, v^{-1}\right]$ is the ring of Laurent polynomials in a variable $v$. Write $q=v^{2}$ and let ${ }^{-}: \mathcal{Z} \rightarrow \mathcal{Z}$ be the ring automorphism satisfying $v^{i} \mapsto v^{-i}$ for all $i$. For $m \in \mathbb{N}$, put

$$
[m]^{!}=[1][2] \cdots[m], \quad \text { where }[i]=\frac{v^{i}-v^{-i}}{v-v^{-1}} .
$$

We also let, for $c \in \mathbb{Z}, t \in \mathbb{N}$,

$$
\left[\begin{array}{l}
c \\
t
\end{array}\right]=\prod_{s=1}^{t} \frac{v^{c-s+1}-v^{-c+s-1}}{v^{s}-v^{-s}} .
$$


If $|c|<t$, then $\left[\begin{array}{l}c \\ t\end{array}\right]=0$, and $\left[\begin{array}{l}c \\ t\end{array}\right]=\frac{[c]^{!}}{[t]^{!}[c-t]^{!}}$for $c \geqslant t \geqslant 0$.

Let $H=H\left(\mathfrak{S}_{r}\right)$ be the Hecke algebra over $\mathcal{Z}$ for the symmetric group $\mathfrak{S}_{r}$. If $S=\{(1,2),(2,3), \cdots,(r-1, r)\}$, then $H$ has $\mathcal{Z}$-basis $T_{w}, w \in \mathfrak{S}_{r}$, and relations

$$
\left\{\begin{array}{l}
T_{s} T_{w}=T_{s w}, \quad l(s w)=1+l(w), \quad s \in S, w \in W ; \\
\left(T_{s}+1\right)\left(T_{s}-q\right)=0, \quad s \in S .
\end{array}\right.
$$

If $V$ is a free $\mathcal{Z}$-module of rank $n$, there is a natural right action of $H$ on $V^{\otimes r}$ by "place" permutations. The $q$-Schur algebra $S_{q}(n, r)$ over $\mathcal{Z}$ is the centralizer ring

$$
S_{q}(n, r)=\operatorname{End}_{H}\left(V^{\otimes r}\right) .
$$

The algebra $S_{q}(n, r)$ is $\mathcal{Z}$-free of rank $\left(n^{2}+r-1\right)$. For more details, see [5], 11]. Put $\mathbf{H}=\mathbb{Q}(v) \otimes H$ and $\mathbf{S}_{q}(n, r)=\mathbb{Q}(v) \otimes S_{q}(n, r)$.

\section{The Quantized enveloping Algebra of $\mathfrak{g l}_{n}$}

The definition below for the quantized enveloping algebra of $\mathfrak{g l}_{n}$ is a slightly modified version of Jimbo [16]; see [23, 3.2], [9, 1.1].

Definition 2.1. The quantized enveloping algebra of $\mathfrak{g l}_{n}$ is the algebra $\mathbf{U}$ over $\mathbb{Q}(v)$ generated by the elements

$$
E_{i}, F_{i} \quad(1 \leqslant i \leqslant n-1), \quad K_{i}, K_{i}^{-1} \quad(1 \leqslant i \leqslant n)
$$

subject to the following relations:

(a) $K_{i} K_{j}=K_{j} K_{i}, \quad K_{i} K_{i}^{-1}=1$;

(b) $K_{i} E_{j}=v^{\epsilon(i, j)} E_{j} K_{i}$, where $\epsilon(i, i)=1, \epsilon(i+1, i)=-1$ and $\epsilon(i, j)=0$, otherwise;

(c) $K_{i} F_{j}=v^{-\epsilon(i, j)} F_{j} K_{i}$ with $\epsilon(i, j)$ as in (b) above;

(d) $E_{i} E_{j}=E_{j} E_{i}, F_{i} F_{j}=F_{j} F_{i}$ when $|i-j|>1$;

(e) $E_{i} F_{j}-F_{j} E_{i}=\delta_{i, j} \frac{\tilde{K}_{i}-\tilde{K}_{i}^{-1}}{v-v^{-1}}$, where $\tilde{K}_{i}=K_{i} K_{i+1}^{-1}$;

(f) $E_{i}^{2} E_{j}-\left(v+v^{-1}\right) E_{i} E_{j} E_{i}+E_{j} E_{i}^{2}=0$ when $|i-j|=1$;

(g) $F_{i}^{2} F_{j}-\left(v+v^{-1}\right) F_{i} F_{j} F_{i}+F_{j} F_{i}^{2}=0$ when $|i-j|=1$.

Relations 2.1(f),(g) are called the quantum Serre relations. The subalgebra generated by the $E_{i}, F_{i}$ and $\tilde{K}_{i}(1 \leqslant i \leqslant n-1)$ is the quantized enveloping algebra $\mathbf{U}_{v}\left(\mathfrak{s l}_{n}\right)$.

There is a unique $\mathbb{Q}$-algebra anti-isomorphism $\Omega: \mathbf{U} \rightarrow \mathbf{U}$ defined by

$$
\Omega\left(E_{i}\right)=F_{i}, \quad \Omega\left(F_{i}\right)=E_{i}, \quad \Omega\left(K_{j}\right)=K_{j}^{-1} \quad \text { and } \quad \Omega(v)=v^{-1} .
$$

Clearly, $\Omega$ extends the anti-isomorphism $\Omega$ defined in [18, 1.2(a)] for $\mathbf{U}_{v}\left(\mathfrak{s l}_{n}\right)$.

Let $\mathbf{U}^{+}$(resp., $\mathbf{U}^{-}, \mathbf{U}^{0}$ ) be the subalgebra of $\mathbf{U}$ generated by the $E_{i}$ (resp., $F_{i}$, $\left.K_{i}\right)$. There is a triangular decomposition $\mathbf{U}^{+} \otimes \mathbf{U}^{0} \otimes \mathbf{U}^{-} \stackrel{\text { mult }}{\longrightarrow} \mathbf{U}^{+} \mathbf{U}^{0} \mathbf{U}^{-}=\mathbf{U}$ which is an isomorphism of vector spaces - see below for references in the integral case. Clearly, the elements $K^{\mathbf{j}}:=K_{1}^{j_{1}} \cdots K_{n}^{j_{n}}$ for all $\mathbf{j}=\left(j_{1}, \cdots, j_{n}\right) \in \mathbb{Z}^{n}$ form a basis for $\mathbf{U}^{0}$. The subalgebras $\mathbf{U}^{+}$and $\mathbf{U}^{-}$are both $\mathbb{N}$-graded in terms of the degrees of monomials in the $E_{i}$ and $F_{i}$. For monomials $M$ in the $E_{i}$ and $M^{\prime}$ in the $F_{i}$, and an element $h \in \mathbf{U}^{0}$, write $\operatorname{deg}\left(M h M^{\prime}\right)=\operatorname{deg}(M)+\operatorname{deg}\left(M^{\prime}\right)$. Observe that deg does not define an algebra grading on $\mathbf{U}$ : the appropriate algebra grading (which we do not use) would be given by $\operatorname{deg}^{\prime}\left(M h M^{\prime}\right)=\operatorname{deg}(M)-\operatorname{deg}\left(M^{\prime}\right)$. 
For an analogue of a Kostant $\mathbb{Z}$-form over $\mathcal{Z}$, define, for $m, t \in \mathbb{N}$ and $c \in \mathbb{Z}$,

$$
E_{i}^{(m)}=\frac{E_{i}^{m}}{[m]^{!}}, \quad F_{i}^{(m)}=\frac{F_{i}^{m}}{[m]^{!}} \quad \text { and } \quad\left[\begin{array}{c}
K_{i} ; c \\
t
\end{array}\right]=\prod_{s=1}^{t} \frac{K_{i} v^{c-s+1}-K_{i}^{-1} v^{-c+s-1}}{v^{s}-v^{-s}} .
$$

Following [23, let $U$ (resp., $U^{+}, U^{-}$) be the $\mathcal{Z}$-subalgebra of $\mathbf{U}$ generated by all $E_{i}^{(m)}, F_{i}^{(m)}, K_{i}$ and $\left[\begin{array}{c}K_{i} ; 0 \\ t\end{array}\right]\left(\right.$ resp., $\left.E_{i}^{(m)}, F_{i}^{(m)}\right)$. Let $U^{0}$ be the $\mathcal{Z}$-subalgebra of $\mathbf{U}$ generated by all $K_{i}$ and $\left[\begin{array}{c}K_{i} ; 0 \\ t\end{array}\right]$. Then $U, U^{+}, U^{0}, U^{-}$are $\mathcal{Z}$-forms for $\mathbf{U}, \mathbf{U}^{+}, \mathbf{U}^{0}$, $\mathbf{U}^{-}$, respectively, and there is a triangular decomposition $U^{+} \otimes U^{0} \otimes U^{-} \cong U^{+} U^{0} U^{-}$ $=U$ as free $\mathcal{Z}$-modules (apply the anti-automorphism $\Omega$ to the triangular decomposition given in [23, (3.2.6)]) 1 ] The following is known from [18, 2.14] and [9, Lemma $2.1]$.

Lemma 2.2. The algebra $U^{0}$ has a $\mathcal{Z}$-basis

$$
K_{1}^{\delta_{1}} \cdots K_{n}^{\delta_{n}}\left[\begin{array}{c}
K_{1} ; 0 \\
t_{1}
\end{array}\right] \cdots\left[\begin{array}{c}
K_{n} ; 0 \\
t_{n}
\end{array}\right], \quad \delta_{i} \in\{0,1\}, t_{i} \in \mathbb{N} .
$$

The formulas below will be useful; see [18, p.269], [17, 4.1(a)].

Lemma 2.3. The following formulas hold in $\mathbf{U}$ :

(1) $\left[\begin{array}{c}K_{i} ; 0 \\ t\end{array}\right]\left[\begin{array}{c}K_{i} ;-t \\ s\end{array}\right]=\left[\begin{array}{c}t+s \\ t\end{array}\right]\left[\begin{array}{c}K_{i} ; 0 \\ t+s\end{array}\right] \quad(t, s \geqslant 0)$;

(2) $\left[\begin{array}{c}K_{i} ; c \\ t\end{array}\right]-v^{-t}\left[\begin{array}{c}K_{i} ; c+1 \\ t\end{array}\right]=-v^{-(c+1)} K_{i}^{-1}\left[\begin{array}{c}K_{i} ; c \\ t-1\end{array}\right] \quad(t \geqslant 1)$;

(3) $\left[\begin{array}{c}K_{i} ;-c \\ t\end{array}\right]=\sum_{0 \leqslant j \leqslant t}(-1)^{j} v^{c(t-j)}\left[\begin{array}{c}c+j-1 \\ j\end{array}\right] K_{i}^{j}\left[\begin{array}{c}K_{i} ; 0 \\ t-j\end{array}\right] \quad(t \geqslant 0, c \geqslant 1)$;

(4) $\left[\begin{array}{c}\left.K_{i} ; c\right] \\ t\end{array}\right]=\sum_{0 \leqslant j \leqslant t} v^{c(t-j)}\left[\begin{array}{c}c \\ j\end{array}\right] K_{i}^{-j}\left[\begin{array}{c}K_{i} ; 0 \\ t-j\end{array}\right] \quad(t \geqslant 0, c \geqslant 0)$;

(5) $E_{i}^{(m)}\left[\begin{array}{c}\left.K_{i} ; c\right] \\ t\end{array}\right]=\left[\begin{array}{c}K_{i} ; c-m \\ t\end{array}\right] E_{i}^{(m)}$ and $E_{i}^{(m)}\left[\begin{array}{c}\left.K_{i+1} ; c\right] \\ t\end{array}\right]=\left[\begin{array}{c}K_{i+1} ; c+m \\ t\end{array}\right] E_{i}^{(m)}$;

(6) $F_{i}^{(m)}\left[\begin{array}{c}\left.K_{i} ; c\right] \\ t\end{array}\right]=\left[\begin{array}{c}K_{i} ; c+m \\ t\end{array}\right] F_{i}^{(m)}$ and $F_{i}^{(m)}\left[\begin{array}{c}K_{i+1} ; c \\ t\end{array}\right]=\left[\begin{array}{c}K_{i+1} ; c-m \\ t\end{array}\right] F_{i}^{(m)}$;

(7) For any positive integers $k, l$, we have

$$
E_{i}^{(k)} F_{i}^{(l)}=\sum_{t=0}^{\min (k, l)} F_{i}^{(l-t)}\left[\begin{array}{c}
\tilde{K}_{i} ; 2 t-k-l \\
t
\end{array}\right] E_{i}^{(k-t)} .
$$

Proof. The formulas (1)-(4) and (7) are proved exactly as in [17]. Finally, (5) and (6) follow from 2.1(b),(c) by induction on $m$.

The commutator formula 2.3(7) plus 2.3(5),(6) show that, for monomials $M$ in the $E_{i}$ and $M^{\prime}$ in the $F_{i}, M M^{\prime}=M^{\prime} M+\sum_{j} M_{j} h_{j} M_{j}^{\prime}$, where $M_{j}$ (resp., $M_{j}^{\prime}$ ) are monomials in the $E_{i}$ 's (resp., $F_{i}$ 's), $h_{j} \in U^{0}$ and

$$
\operatorname{deg}\left(M_{j} h_{j} M_{j}^{\prime}\right) \leqslant \operatorname{deg}\left(M M^{\prime}\right)-2 .
$$

\footnotetext{
${ }^{1}$ Note that the Lusztig $\mathcal{Z}$-form ${ }^{\prime} U$ of $\mathbf{U}_{v}\left(\mathfrak{s l}_{n}\right)$ is generated by all $E_{i}^{(m)}, F_{i}^{(m)}, \tilde{K}_{i}$, and $\tilde{K}_{i}^{-1}$. Thus, by 9 2.6], the $\mathcal{Z}$-form $U$ can be generated by all $E_{i}^{(m)}, F_{i}^{(m)}, \tilde{K}_{i}, \tilde{K}_{i}^{-1}, K_{1}$ and $\left[\begin{array}{c}K_{1} ; 0 \\ t\end{array}\right]$.
} 


\section{The Beilinson-Lusztig-MacPherson construction AND MONOMIAL BASES}

Let $\widetilde{\Xi}$ be the set of all $n \times n$ matrices over $\mathbb{Z}$ with all off-diagonal entries in $\mathbb{N}$, and let $\Xi=M_{n}(\mathbb{N})$ be the subset of $\widetilde{\Xi}$ consisting of matrices with entries all in $\mathbb{N}$. Let $\sigma: \Xi \rightarrow \mathbb{N}$ be the map sending a matrix to the sum of its entries. Then, for $r \in \mathbb{N}$, the inverse image $\Xi_{r}:=\sigma^{-1}(r)$ is the set of $n \times n$ matrices in $\Xi$ whose entries sum to $r$. For $1 \leqslant i, j \leqslant n$, let $E_{i, j} \in \Xi$ be the matrix $\left(a_{k, l}\right)$ with $a_{k, l}=\delta_{i, k} \delta_{j, l}$.

Let $U_{r}$ be the algebra over $\mathcal{Z}$ introduced in $[1,1.2]^{2}$ It has a normalized $\mathcal{Z}$-basis $\{[A]\}_{A \in \Xi_{r}}$ defined in [1 1.4]. In particular, if $\lambda \in \mathbb{N}^{n}$ with $D=\operatorname{diag}(\lambda) \in \Xi_{r}$, then (cf. [1, 1.3])

$$
[D][A]=\left\{\begin{array}{ll}
{[A],} & \text { if } \lambda=\operatorname{ro}(A) \\
0, & \text { otherwise } ;
\end{array} \text { and }[A][D]= \begin{cases}{[A],} & \text { if } \lambda=\operatorname{co}(A) \\
0, & \text { otherwise }\end{cases}\right.
$$

where $\operatorname{ro}(A)=\left(\sum_{j} a_{1, j}, \cdots, \sum_{j} a_{n, j}\right)$ and $c o(A)=\left(\sum_{i} a_{i, 1}, \cdots, \sum_{i} a_{i, n}\right)$ are the sequences of row and column sums of $A=\left(a_{i, j}\right)$. We put $\mathbf{U}_{r}=\mathbb{Q}(v) \otimes_{\mathcal{Z}} U_{r}$.

In [9] 1.4], the algebra $U_{r}$ is shown to be naturally isomorphic to the $q$-Schur algebra $S_{q}(n, r)$ as defined in (1.0.2). In the sequel, we often call $U_{r}$ and $\mathbf{U}_{r} q$-Schur algebras 3

Let $\mathbf{K}$ be the $\mathcal{Z}$-algebra (without 1), defined in [1, §4], with basis $\{[A]\}_{A \in \widetilde{\Xi}}$, and let $\dot{\mathbf{U}}=\mathbb{Q}(v) \otimes_{\mathcal{Z}} \mathbf{K}$ 目 The multiplication $\cdot$ in $\mathbf{K}$ (and hence in $\dot{\mathbf{U}}$ ) is defined in [1, 4.4] by specializing $v^{\prime}$ to 1 from another algebra over $\mathbb{Q}(v)\left[v^{\prime}, v^{\prime-1}\right]$ whose multiplication is induced from the stabilization property of the multiplication of $q$-Schur algebras. By the definition in 1, 4.5], the relations (3.0.1), $D \in \tilde{\Xi}$, continue to hold in $\dot{\mathbf{U}}$.

As in [1, 5.1], $\dot{\mathbf{U}}_{\infty}$ is the vector space of all formal (possibly infinite) $\mathbb{Q}(v)$-linear combinations $\sum_{A \in \widetilde{\Xi}} \beta_{A}[A]$ satisfying: for diagonal $D, D^{\prime} \in \widetilde{\Xi}$, the sums

$$
\sum_{A \in \widetilde{\Xi}} \beta_{A}[D] \cdot[A] \quad \text { and } \quad \sum_{A \in \widetilde{\Xi}} \beta_{A}[A] \cdot\left[D^{\prime}\right]
$$

are finite. Defining the product of $\sum_{A \in \widetilde{\Xi}} \beta_{A}[A], \sum_{B \in \widetilde{\Xi}} \gamma_{B}[B] \in \dot{\mathbf{U}}_{\infty}$ to be

$$
\sum_{A, B} \beta_{A} \gamma_{B}[A] \cdot[B]
$$

gives $\dot{\mathbf{U}}_{\infty}$ an associative algebra structure, with $1=\sum[D]$, the sum over all diagonal $D \in \widetilde{\Xi}$. Also, $\dot{\mathbf{U}}$ is naturally a subalgebra (without 1 ) of $\dot{\mathbf{U}}_{\infty}$.

\footnotetext{
${ }^{2}$ The algebra $U_{r}$ is denoted $\mathbf{K}_{r}$ in [1].

${ }^{3}$ The algebra $U_{r}$ can be roughly described as follows: Let $G=G L_{r}\left(p^{d}\right)$ for some prime power $p^{d}$. Let $\mathcal{S}_{q}(n, r)=\mathbb{C} \otimes S_{q}(n, r)$ via the base change $\mathcal{Z} \rightarrow \mathbb{C}, v \mapsto p^{d / 2}$. It is well known (see, e.g., [5. (2.24]) that

$$
\mathcal{S}_{q}(n, r) \cong \operatorname{End}_{G}\left(\bigoplus \operatorname{ind}_{P_{\lambda}}^{G} \mathbb{C}\right)
$$

here $\lambda$ runs over all compositions $\lambda$ of $r$ into $n$ parts, and $P_{\lambda}$ denotes the corresponding parabolic subgroup of $G$. Using the geometry of relative positions of pairs of $n$-step filtrations on $r$-dimensional space, [1] defines $U_{r}$ directly as a kind of "deformation" of (3.0.2).

${ }^{4}$ The notation here has been abused as in $[1]$ : the basis $\{[A]\}_{A \in \Xi_{r}}$ for a $q$-Schur algebra is not

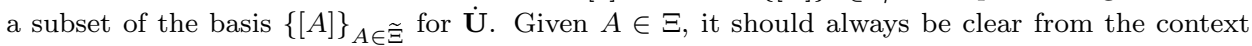
whether $[A]$ is to be regarded as a basis element of $\mathbf{U}$ or of $\mathbf{U}_{r}$.
} 
Let $\Xi^{ \pm}$be the set of all $A \in \Xi$ whose diagonal entries are zero. Given $r \in \mathbb{N}$, $r>0, A \in \Xi^{ \pm}$and $\mathbf{j}=\left(j_{1}, \cdots, j_{n}\right) \in \mathbb{Z}^{n}$, we define

$$
\begin{aligned}
& A(\mathbf{j}, r)=\sum_{\substack{D \in \Xi^{0} \\
\sigma(A+D)=r}} v^{\sum_{i} d_{i} j_{i}}[A+D] \in \mathbf{U}_{r}, \\
& A(\mathbf{j})=A(\mathbf{j}, \infty)=\sum_{D \in \widetilde{\Xi}^{0}} v^{\sum_{i} d_{i} j_{i}}[A+D] \in \dot{\mathbf{U}}_{\infty},
\end{aligned}
$$

where $\Xi^{0}$ (resp., $\widetilde{\Xi}^{0}$ ) denotes the subset of diagonal matrices in $\Xi$ (resp., $\widetilde{\Xi}$ ) and $D=\operatorname{diag}\left(d_{1}, \cdots, d_{n}\right)$. If $\sigma(A)>r$, then $A(\mathbf{j}, r)=0$. For any diagonal matrix $D \in \Xi_{r}$, we have from (3.0.1)

$$
A(\mathbf{0}, r)[D]= \begin{cases}{\left[A+D^{\prime}\right],} & \text { if } \operatorname{co}\left(D^{\prime}\right)=\operatorname{ro}(D)-\operatorname{co}(A) \in \mathbb{N}^{n} \\ 0, & \text { if such a } D^{\prime} \in \mathbb{N}^{n} \text { does not exist. }\end{cases}
$$

Of course, $\mathbf{0}:=(0, \cdots, 0) \in \mathbb{N}^{n}$. Obviously, the $D^{\prime}$ in (3.0.3) satisfying $\operatorname{co}\left(D^{\prime}\right)=$ $\operatorname{ro}(D)-\operatorname{co}(A)$ is unique, if it exists.

Let $\mathbf{V}$ be the subspace of $\dot{\mathbf{U}}_{\infty}$ spanned by

$$
\mathcal{B}=\left\{A(\mathbf{j}) \mid A \in \Xi^{ \pm}, \mathbf{j} \in \mathbb{Z}^{n}\right\} .
$$

The next result is proved in [1, 5.5, 5.7].

Proposition 3.1. (1) V is a subalgebra of $\dot{\mathbf{U}}_{\infty}$ with $\mathbb{Q}(v)$-basis $\mathcal{B}$. It is generated by $E_{h, h+1}(\mathbf{0}), E_{h+1, h}(\mathbf{0})$ and $0(\mathbf{j})$ for all $1 \leqslant h<n$ and $\mathbf{j} \in \mathbb{Z}^{n}$.

(2) For any positive integer $r$, the $q$-Schur algebra $\mathbf{U}_{r}$ is generated by the elements

$$
E_{h, h+1}(\mathbf{0}, r), \quad E_{h+1, h}(\mathbf{0}, r), \quad \text { and } \quad 0(\mathbf{j}, r)
$$

for all $1 \leqslant h<n$ and $\mathbf{j} \in \mathbb{N}^{n}$.

(3) There is an algebra isomorphism $\mathbf{U} \stackrel{\sim}{\rightarrow} \mathbf{V}$ satisfying

$$
E_{h} \mapsto E_{h, h+1}(\mathbf{0}), \quad K^{\mathbf{j}} \mapsto 0(\mathbf{j}), \quad F_{h} \mapsto E_{h+1, h}(\mathbf{0})
$$

and an algebra epimorphism $\zeta_{r}: \mathbf{U} \rightarrow \mathbf{U}_{r}$ satisfying

$$
E_{h} \mapsto E_{h, h+1}(\mathbf{0}, r), \quad K^{\mathbf{j}} \mapsto 0(\mathbf{j}, r), \quad F_{h} \mapsto E_{h+1, h}(\mathbf{0}, r) .
$$

We shall identify $\mathbf{U}$ with $\mathbf{V}$ and hence identify $E_{h}$ with $E_{h, h+1}(\mathbf{0})$, etc., in the sequel. We now describe a monomial basis for $\mathbf{U}$.

Let $\Xi^{+}$(resp., $\Xi^{-}$) be the subset of $\Xi$ consisting of those matrices $\left(a_{i, j}\right)$ with $a_{i, j}=0$ for all $i \geqslant j$ (resp., $i \leqslant j$ ). For $A \in \Xi$, write $A=A^{+}+A^{0}+A^{-}$with $A^{+} \in \Xi^{+}, A^{0} \in \Xi^{0}$, and $A^{-} \in \Xi^{-}$. We also introduce the degree function 5

$$
\operatorname{deg}(A)=\sum_{1 \leqslant i, j \leqslant n}|j-i| a_{i, j}
$$

Let $A=\left(a_{i j}\right) \in \Xi$. For $i<j$, let $\sigma_{i, j}(A)=\sum_{s \leqslant i ; t \geqslant j} a_{s, t}$ and $\sigma_{j, i}(A)=$ $\sum_{s \leqslant i ; t \geqslant j} a_{t, s}$. Define, following [1, 3.5], $A^{\prime} \preccurlyeq A$ if and only if $\sigma_{i, j}\left(A^{\prime}\right) \leqslant \sigma_{i, j}(A)$ and $\sigma_{j, i}\left(A^{\prime}\right) \leqslant \sigma_{j, i}(A)$ for all $1 \leqslant i<j \leqslant n$. Put $A^{\prime} \prec A$ if $A^{\prime} \preccurlyeq A$ and, for some pair $(i, j)$ with $i<j$, either $\sigma_{i, j}\left(A^{\prime}\right)<\sigma_{i, j}(A)$ or $\sigma_{j, i}\left(A^{\prime}\right)<\sigma_{j, i}(A)$. Since $\operatorname{deg} A^{+}=\sum_{i=1}^{n-1} \sigma_{i, i+1}(A)$, and $\operatorname{deg} A^{-}=\sum_{i=1}^{n-1} \sigma_{i+1, i}(A)$, the lemma below holds.

\footnotetext{
${ }^{5}$ The degree function here differs from the $\Psi$ function [1, p.668]. But, it plays a similar role for induction; see 4.14 .
} 
Lemma 3.2. If $A^{\prime} \preccurlyeq A$, then $\operatorname{deg}\left(A^{\prime}\right) \leqslant \operatorname{deg}(A)$.

Note that $A^{\prime} \prec A$ does not necessarily imply that $\operatorname{deg}\left(A^{\prime}\right)<\operatorname{deg}(A)$.

For $A \in \Xi^{ \pm}$and $\mathbf{j} \in \mathbb{Z}^{n}$, let

$$
M^{(A, \mathbf{j})}=E^{\left(A^{+}\right)} \cdot 0(\mathbf{j}) \cdot F^{\left(A^{-}\right)},
$$

where

$$
E^{\left(A^{+}\right)}=\prod_{1 \leqslant i \leqslant h<j \leqslant n} E_{h}^{\left(a_{i, j}\right)} \text { and } F^{\left(A^{-}\right)}=\prod_{1 \leqslant j \leqslant h<i \leqslant n} F_{h}^{\left(a_{i, j}\right)} .
$$

The orders in which the products $E^{\left(A^{+}\right)}$and $F^{\left(A^{-}\right)}$are taken are defined as follows: For the $j$ th column (reading upwards) $a_{j-1, j}, \cdots, a_{1, j}(2 \leqslant j \leqslant n)$ of $A^{+}$, fix the following reduced expression for the longest word $w_{0, j}$ of $\mathfrak{S}_{j}$ :

$$
\begin{aligned}
w_{0, j} & =s_{j-1}\left(s_{j-2} s_{j-1}\right)\left(s_{j-3} s_{j-2} s_{j-1}\right) \cdots\left(s_{1} s_{2} \cdots s_{j-1}\right) \\
& =\left(s_{j-1} \cdots s_{1}\right)\left(s_{j-1} \cdots s_{2}\right) \cdots\left(s_{j-1} s_{j-2}\right) s_{j-1} .
\end{aligned}
$$

Here (and later), $s_{i}=(i, i+1)$ for $1 \leqslant i<j$. Put

$$
M_{j}=M_{j}\left(A^{+}\right)=E_{j-1}^{\left(a_{j-1, j}\right)}\left(E_{j-2}^{\left(a_{j-2, j}\right)} E_{j-1}^{\left(a_{j-2, j}\right)}\right) \cdots\left(E_{1}^{\left(a_{1, j}\right)} E_{2}^{\left(a_{1, j}\right)} \cdots E_{j-1}^{\left(a_{1, j}\right)}\right) .
$$

Similarly, for the $j$ th row (reading to the right) $a_{j, 1}, \cdots, a_{j, j-1}(2 \leqslant j \leqslant n)$ of $A^{-}$, put

$$
M_{j}^{\prime}=\left(F_{j-1}^{\left(a_{j, 1}\right)} \cdots F_{2}^{\left(a_{j, 1}\right)} F_{1}^{\left(a_{j, 1}\right)}\right) \cdots\left(F_{j-1}^{\left(a_{j, j-2}\right)} F_{j-2}^{\left(a_{j, j-2}\right)}\right) F_{j-1}^{\left(a_{j, j-1}\right)}=\Omega\left(M_{j}\left(A^{\prime}\right)\right)
$$

(cf. (2.1.1)), where $A^{\prime}=\left(A^{-}\right)^{T}$ is the transpose of $A^{-}$. Then we have $E^{\left(A^{+}\right)}=$ $M_{n} M_{n-1} \cdots M_{2}$ and $F^{\left(A^{-}\right)}=M_{2}^{\prime} M_{3}^{\prime} \cdots M_{n}^{\prime}$. Clearly, $\operatorname{deg} E^{\left(A^{+}\right)}=\operatorname{deg}\left(A^{+}\right)$and $\operatorname{deg} F^{\left(A^{-}\right)}=\operatorname{deg}\left(A^{-}\right)$. The following result is also essentially proved in [1].

Proposition 3.3. The set

$$
\mathcal{M}=\left\{M^{(A, \mathbf{j})} \mid A \in \Xi^{ \pm}, \mathbf{j} \in \mathbb{Z}^{n}\right\}
$$

forms a basis for $\mathbf{U}$. For $A \in \Xi^{ \pm}, \mathbf{j} \in \mathbb{Z}^{n}$, there exist $a \in \mathbb{Z}, f_{\mathbf{j}^{\prime}, B}, g_{\mathbf{j}^{\prime \prime}, C} \in \mathbb{Q}(v)$ such that

$$
\begin{aligned}
M^{(A, \mathbf{j})} & =v^{a} A(\mathbf{j})+\sum_{\substack{\mathbf{j}^{\prime} \in \mathbb{Z}^{n}, B \in \Xi^{ \pm} \\
B \prec A}} f_{\mathbf{j}^{\prime}, B} B\left(\mathbf{j}^{\prime}\right), \\
A(\mathbf{j}) & =v^{-a} M^{(A, \mathbf{j})}+\sum_{\substack{\mathbf{j}^{\prime \prime} \in \mathbb{Z}^{n}, C \in \Xi^{ \pm} \\
C \prec A}} g_{\mathbf{j}^{\prime \prime}, C} M^{\left(C, \mathbf{j}^{\prime \prime}\right)} .
\end{aligned}
$$

In particular, the set $\left\{E^{(A)}\right\}_{A \in \Xi^{+}}$(resp., $\left\{F^{(B)}\right\}_{B \in \Xi^{-}}$) forms a basis for $\mathbf{U}^{+}$(resp., $\left.\mathbf{U}^{-}\right)$.

\footnotetext{
${ }^{6}$ Observe that changing from the first reduced expression in 3.2 .1 to the second requires only relations of the form $s_{i} s_{j}=s_{j} s_{i},|i-j|>1$. Thus, by definition 2.1 we have

$$
M_{j}=\left(E_{j-1}^{\left(a_{j-1, j}\right)} \cdots E_{1}^{\left(a_{1, j}\right)}\right)\left(E_{j-2}^{\left(a_{j-2, j}\right)} \cdots E_{2}^{\left(a_{1, j}\right)}\right) \cdots\left(E_{j-1}^{\left(a_{2, j}\right)} E_{j-2}^{\left(a_{1, j}\right)}\right) E_{j-1}^{\left(a_{1, j}\right)} .
$$
}

This is the original definition given in $[1]$. 
Proof. For the first assertion, see [1, 5.7]. Next, 1, 5.4(c)] (and the discussion following it) implies (3.3.1), while (3.3.2) is obtained by solving (3.3.1) inductively. The final assertion follows from an argument along the line of that of [1,5.5] (see [12, 4.3] for some details).

The basis $\mathcal{M}$ is the monomial basis associated to the given ordering on the $w_{0, j}$ above.

Corollary 3.4. Let $A \in \Xi^{+}$and let $M$ be any monomial in the $E_{i}$. Then the product $M E^{(A)}$ is a (finite) $\mathcal{Z}$-linear combination of $E^{(B)}$ with $B \in \Xi^{+}$and $\operatorname{deg}(B) \leqslant$ $\operatorname{deg}(M)+\operatorname{deg}(A)$. Thus, $M$ itself can be written as a linear combination of $E^{(B)}$ with $B \in \Xi^{+}$and $\operatorname{deg}(B) \leqslant \operatorname{deg}(M)$. A similar statement holds for the $F_{i}$.

Proof. Using [1 5.4(c)] again, we have

$$
E^{(A)}=M^{(A, \mathbf{0})}=A(\mathbf{0})+\sum_{\substack{\mathbf{j} \in \mathbb{Z}^{n}, B^{\prime} \in \Xi^{ \pm} \\ B^{\prime} \prec A}} f_{\mathbf{j}, B^{\prime}} B^{\prime}(\mathbf{j}) .
$$

The first formula given in [1 5.3] implies that, if $A^{\prime} \in \Xi^{+}$, then $E_{h} A^{\prime}(\mathbf{0})$ is a linear combination of terms $B(\mathbf{0})$ with $B \in \Xi^{+}$. (The fact that $A^{\prime} \in \Xi^{+}$is essential to guarantee that the summands in [1 5.3] of the form $B(\mathbf{j})$ with $\mathbf{j} \neq \mathbf{0}$ all have zero coefficient.) Since $E^{(A)}$ is a product (up to a scalar) of various $E_{h}=E_{h, h+1}(\mathbf{0})$, induction shows that the only $B^{\prime}(\mathbf{j})$ that occur in (3.4.1) are those with $B^{\prime} \in \Xi^{+}$ and $\mathbf{j}=\mathbf{0}$. Therefore, we obtain

$$
E^{(A)}=A(\mathbf{0})+\sum_{B^{\prime} \in \Xi^{+}: B^{\prime} \prec A} f_{\mathbf{0}, B^{\prime}} B^{\prime}(\mathbf{0}) .
$$

Clearly, if $f_{\mathbf{0}, B^{\prime}} \neq 0$, then $B^{\prime} \prec A$ implies $\operatorname{deg}\left(B^{\prime}\right) \leqslant \operatorname{deg}(A)$, by 3.2 and $B^{\prime} \in \Xi^{+}$ as well.

To prove the corollary, we can easily reduce to the special case when $M=E_{h}$ for $1 \leqslant h \leqslant n-1$. Applying [1, 5.3, p. 672] again shows that the product $E_{h} A(\mathbf{0})$ (resp., $\left.E_{h} B^{\prime}(\mathbf{0})\right)$ is a linear combination of $B^{\prime \prime}(\mathbf{0})$ with $B^{\prime \prime} \in \Xi^{+}, \operatorname{deg}\left(B^{\prime \prime}\right) \leqslant \operatorname{deg}(A)+1$ (resp., $\left.\operatorname{deg}\left(B^{\prime \prime}\right) \leqslant \operatorname{deg}\left(B^{\prime}\right)+1 \leqslant \operatorname{deg}(A)+1\right)$. Every $B^{\prime \prime}(\mathbf{0})$ is a linear combination of $E^{(B)}$ with $B \in \Xi^{+}, B \preccurlyeq B^{\prime \prime}$ by (3.4.2). So $E_{h} E^{(A)}$ is a linear combination of $E^{(B)}$ with $B \in \Xi^{+}$and $\operatorname{deg}(B) \leqslant \operatorname{deg}\left(B^{\prime \prime}\right) \leqslant \operatorname{deg}(A)+1$.

Remark 3.5. We note that the inequalities $\operatorname{deg}(B) \leqslant \operatorname{deg}(A)+\operatorname{deg}(M)$ in the statement of 3.4 can even be replaced by the equalities $\operatorname{deg}(B)=\operatorname{deg}(A)+\operatorname{deg}(M)$, using the fact that the relations defining $\mathbf{U}^{+}$are homogeneous and the monomial basis given in the last assertion of 3.3 preserves the graded structure on $\mathbf{U}^{+}$. We thank Fu Qiang for pointing this out.

\section{The Algebra $\mathbf{S}_{r}$}

Let $X$ be another indeterminate which is independent of $v$. For $t \in \mathbb{N}$, put

$$
[X ; t]^{!}=(X-1)(X-v) \cdots\left(X-v^{t-1}\right),
$$

with $[X ; 0]^{!}=1$ by definition.

\footnotetext{
${ }^{7}$ We will make much use of this result, which holds for both $\mathbf{U}$ and for $\mathbf{U}_{r}$, in the sequel.
} 
Definition 4.1. Let $\mathbf{S}_{r}$ be the associative algebra over $\mathbb{Q}(v)$ generated by the elements

$$
\mathrm{e}_{i}, \mathbf{f}_{i}, \mathrm{k}_{i} \quad(1 \leqslant i \leqslant n-1)
$$

subject to the relations:

(a) $\mathrm{k}_{i} \mathrm{k}_{j}=\mathrm{k}_{j} \mathrm{k}_{i}$;

(b) $\left[\mathrm{k}_{1} ; t_{1}\right]^{!}\left[\mathrm{k}_{2} ; t_{2}\right]^{!} \cdots\left[\mathrm{k}_{n-1} ; t_{n-1}\right]^{!}=0 \forall t_{i} \in \mathbb{N}$ such that $t_{1}+\cdots+t_{n-1}=r+1$;

(c) $\mathrm{e}_{i} \mathrm{e}_{j}=\mathrm{e}_{j} \mathrm{e}_{i}, \mathrm{f}_{i} \mathrm{f}_{j}=\mathbf{f}_{j} \mathrm{f}_{i} \quad(|i-j|>1)$;

(d) $\mathrm{e}_{i}^{2} \mathrm{e}_{j}-\left(v+v^{-1}\right) \mathrm{e}_{i} \mathrm{e}_{j} \mathrm{e}_{i}+\mathrm{e}_{j} \mathrm{e}_{i}^{2}=0 \quad(|i-j|=1)$;

(e) $\mathbf{f}_{i}^{2} \mathbf{f}_{j}-\left(v+v^{-1}\right) \mathbf{f}_{i} \mathbf{f}_{j} \mathbf{f}_{i}+\mathbf{f}_{j} \mathbf{f}_{i}^{2}=0 \quad(|i-j|=1)$;

(f) $\mathrm{k}_{i} \mathrm{e}_{j}=v^{\epsilon(i, j)} \mathbf{e}_{j} \mathrm{k}_{i}, \quad \mathbf{k}_{i} \mathbf{f}_{j}=v^{-\epsilon(i, j)} \mathbf{f}_{j} \mathrm{k}_{i}$ with $\epsilon(i, j)$ as in 2.1(b);

(g) $\mathrm{e}_{i} \mathbf{f}_{j}-\mathbf{f}_{j} \mathrm{e}_{i}=\delta_{i, j} \frac{\tilde{\mathrm{k}}_{i}-\tilde{\mathrm{k}}_{i}^{-1}}{v-v^{-1}}$, where $\tilde{\mathrm{k}}_{i}=\mathrm{k}_{i} \mathrm{k}_{i+1}^{-1}, 1 \leqslant i \leqslant n-1$, with $\mathrm{k}_{n}=$ $v^{r} \mathrm{k}_{1}^{-1} \cdots \mathrm{k}_{n-1}^{-1}$.

Since $\left[\mathrm{k}_{i} ; t_{i}\right]^{!}=0$ if $t_{i}=r+1$, each $\mathrm{k}_{i}$ is invertible and $\mathrm{k}_{i}^{-1}$ is a polynomial of $\mathrm{k}_{i}$ of degree $r$; so the definitions of $\tilde{\mathrm{k}}_{i}$ and $\mathrm{k}_{n}$ make sense. Also, 4.1(f) holds for $i=n$. By 2.1, there is a surjective homomorphism $\mathbf{U} \rightarrow \mathbf{S}_{r}$ in which $E_{i} \mapsto \mathrm{e}_{i}, F_{i} \mapsto \mathbf{f}_{i}$ and $K_{j} \mapsto \mathrm{k}_{j}$. In particular, for $A \in \Xi^{+}$, let $\mathrm{e}^{(A)}$ be the image of $E^{(A)}$ under this homomorphism, with a similar convention for $\mathrm{f}^{(A)}, A \in \Xi^{-}$. The relations in [2.3] hold with $E_{i}, F_{i}, K_{i}$ replaced by $\mathrm{e}_{i}, \mathrm{f}_{i}, \mathrm{k}_{i}$, respectively. The definition implies the following result.

Lemma 4.2. (1) There is a unique $\mathbb{Q}(v)$-algebra anti-automorphism 8 on $\mathbf{S}_{r}$ satisfying

$$
\tau\left(\mathrm{e}_{i}\right)=\mathrm{f}_{i}, \quad \tau\left(\mathrm{f}_{i}\right)=\mathrm{e}_{i}, \quad \tau\left(\mathrm{k}_{i}\right)=\mathrm{k}_{i} .
$$

(2) There is a unique $\mathbb{Q}(v)$-algebra anti-automorphism $\gamma$ on $\mathbf{S}_{r}$ satisfying

$$
\gamma\left(\mathrm{e}_{i}\right)=\mathrm{e}_{n-i}, \quad \gamma\left(\mathbf{f}_{i}\right)=\mathbf{f}_{n-i}, \quad \gamma\left(\mathrm{k}_{i}\right)=\mathrm{k}_{n-i+1} .
$$

(3) There is a unique $\mathbb{Q}$-algebra involution ${ }^{-}$on $\mathbf{S}_{r}$ satisfying

$$
\overline{\mathrm{e}}_{i}=\mathrm{e}_{i}, \quad \overline{\mathbf{f}}_{i}=\mathrm{f}_{i}, \quad \overline{\mathrm{k}}_{i}=\mathrm{k}_{i}^{-1}, \quad \bar{v}=v^{-1} .
$$

Proposition 4.3. Let $\mathbf{t}=\left(t_{1}, \cdots, t_{n}\right) \in \mathbb{N}^{n}$ and put $|\mathbf{t}|=t_{1}+\cdots+t_{n}$. Then

$$
[\mathrm{k} ; \mathbf{t}]:=\left[\mathrm{k}_{1} ; t_{1}\right]^{!}\left[\mathrm{k}_{2} ; t_{2}\right]^{!} \cdots\left[\mathrm{k}_{n} ; t_{n}\right]^{!}=0 \quad \text { whenever }|\mathbf{t}|=r+1 \text {. }
$$

In particular, $\left[\mathrm{k}_{n} ; r+1\right]^{!}=0$.

Proof. If $t_{n}=0$, the result holds by 4.1(b). So assume $t_{n} \geqslant 1$. Then

$$
\begin{aligned}
& {\left[\mathrm{k}_{n} ; t_{n}\right]^{!}=-v^{t_{n}-1} \mathrm{k}_{1}^{-1} \cdots \mathrm{k}_{n-1}^{-1}\left[\mathrm{k}_{n} ; t_{n}-1\right]^{!}\left(\mathrm{k}_{1} \cdots \mathrm{k}_{n-1}-v^{r-t_{n}+1}\right)} \\
& \quad=-v^{t_{n}-1} \mathrm{k}_{1}^{-1} \cdots \mathrm{k}_{n-1}^{-1}\left[\mathrm{k}_{n} ; t_{n}-1\right]^{!} \sum_{i=1}^{n-1} v^{t_{1}+\cdots+t_{i-1}}\left(\mathrm{k}_{i}-v^{t_{i}}\right) \mathrm{k}_{i+1} \cdots \mathrm{k}_{n-1} .
\end{aligned}
$$

(Observe the above sum is telescoping.) Putting $a_{i}=-v^{t_{1}+\cdots+t_{i-1}+t_{n-1}-1} \mathrm{k}_{1}^{-1} \cdots$ $\mathrm{k}_{i}^{-1}$

$$
[\mathrm{k} ; \mathbf{t}]=\sum_{i=1}^{n-1} a_{i}\left[\mathrm{k}_{1} ; t_{1}\right]^{!} \cdots\left[\mathrm{k}_{i} ; t_{i}+1\right]^{!} \cdots\left[\mathrm{k}_{n-1} ; t_{n-1}\right]^{!}\left[\mathrm{k}_{n} ; t_{n}-1\right]^{!}=0
$$

\footnotetext{
${ }^{8}$ The composition of this isomorphism and the bar involution - below is the "little" version of the map $\Omega$ defined in 2.1.1.
} 
by induction on $t_{n}$.

Remarks 4.4. (1) 4.3 provides a connection between the presentation 4.1 and that given in [8]. Let $\mathbf{S}_{r}^{0}$ be the commutative subalgebra of $\mathbf{S}_{r}$ generated by $\mathrm{k}_{1}, \cdots, \mathrm{k}_{n-1}$. By 4.7 below, the relations 4.1 (a), (b) provide a presentation for $\mathbf{S}_{r}^{0}$. However, by 4.3 and 4.6 below, $\mathbf{S}_{r}^{0}$ can also be described differently, taking generators $\mathrm{k}_{1}, \cdots, \mathrm{k}_{n}$ satisfying the relations $\mathrm{k}_{i} \mathrm{k}_{j}=\mathrm{k}_{j} \mathrm{k}_{i},\left[\mathrm{k}_{i} ; r+1\right]^{!}=0$, and $\mathrm{k}_{1} \cdots \mathrm{k}_{n}=v^{r}$; see [8] Prop. 7.4]. This gives another presentation of $\mathbf{S}_{r}^{0}$, replacing $\mathrm{k}_{1}, \cdots, \mathrm{k}_{n-1}$ by new generators $\mathrm{k}_{1}, \cdots, \mathrm{k}_{n}$ and replacing the relations 4.1(a),(b) by those above. This presentation is studied in [8; it has fewer relations than 4.3] but one more generator $\mathrm{k}_{n}$ and the relations involving $\mathrm{k}_{n}$.

(2) To justify the relations for $\mathbf{S}_{r}^{0}$, let $L_{v}(\lambda)$ be the irreducible type $1 \mathbf{U}$-module with highest weight $\lambda$, where $\lambda$ is a partition of $r$ with at most $n$ parts. Suppose $u_{\mu} \in L_{v}(\lambda)_{\mu}$, the $\mu$-weight space of $L_{v}(\lambda)$. Then $\mu \in \mathbb{N}^{n},|\mu|=r, \mu \unlhd \lambda$ (the dominance order - see below the proof of Prop. 4.5) and $K_{i} u_{\mu}=v^{\mu_{i}} u_{\mu}$. Thus,

$$
\prod_{i=1}^{n}\left[K_{i} ; t_{i}\right]^{!} u_{\mu}=\prod_{i=1}^{n}\left[v^{\mu_{i}} ; t_{i}\right]^{!} u_{\mu}
$$

Since $\prod_{i=1}^{n}\left[v^{\mu_{i}} ; t_{i}\right]^{!}=0$ whenever $t_{1}+\cdots+t_{n}=r+1, L_{v}(\lambda)$ naturally becomes an $\mathbf{S}_{r}$-module.

By definition, for $\mathbf{t}=\left(t_{1}, \cdots, t_{n}\right) \in \mathbb{N}^{n}$ and $A \in \Xi$, $\mathrm{e}^{\left(A^{+}\right)}, \mathrm{f}^{\left(A^{-}\right)}$are the images of $E^{\left(A^{+}\right)}, F^{\left(A^{-}\right)}$, respectively, under the epimorphism $\mathbf{U} \rightarrow \mathbf{S}_{r}$. Thus,

$$
\begin{gathered}
\mathrm{e}^{\left(A^{+}\right)}=\prod_{1 \leqslant i \leqslant h<j \leqslant n} \mathrm{e}_{h}^{\left(a_{i, j}\right)}, \quad \mathrm{f}^{\left(A^{-}\right)}=\prod_{1 \leqslant j \leqslant h<i \leqslant n} \mathrm{f}_{h}^{\left(a_{i, j}\right)}, \\
\mathrm{k}_{\mathbf{t}}=\prod_{i=1}^{n}\left[\begin{array}{c}
\mathrm{k}_{i} ; 0 \\
t_{i}
\end{array}\right] .
\end{gathered}
$$

Here the order in the products is the same as the order used for $E^{\left(A^{+}\right)}$and $F^{\left(A^{-}\right)}$ in $\S 3$. The next result is a direct consequence of the defining relations on the $\mathrm{k}_{i}$ (cf. [8, 7.4(c), 7.6(a)]).

Lemma 4.5. Let $\mathbf{t}=\left(t_{1}, \cdots, t_{n}\right) \in \mathbb{N}^{n}$. Then:

(1) $\mathrm{k}_{\mathbf{t}}=0$ if $|\mathbf{t}|>r$.

(2) If $|\mathbf{t}|=r$, then $\mathbf{k}_{i} \mathrm{k}_{\mathbf{t}}=v^{t_{i}} \mathbf{k}_{\mathbf{t}}$; in particular, $\left[\begin{array}{c}\mathrm{k}_{i} ; c \\ t\end{array}\right] \mathrm{k}_{\mathbf{t}}=\left[\begin{array}{c}t_{i}+c \\ t\end{array}\right] \mathrm{k}_{\mathbf{t}}$.

Proof. To see (1), observe that

$$
\left[\begin{array}{c}
\mathrm{k}_{i} ; 0 \\
t_{i}
\end{array}\right]=\prod_{s=1}^{t_{i}} \frac{\mathrm{k}_{i} v^{-s+1}-\mathrm{k}_{i}^{-1} v^{+s-1}}{v^{s}-v^{-s}}=\prod_{s=1}^{t_{i}} \frac{\mathrm{k}_{i}^{-1} v^{-s+1}\left(\mathrm{k}_{i}^{2}-v^{2(s-1)}\right)}{v^{s}-v^{-s}}
$$

so that $\left[\mathrm{k}_{i}, t_{i}\right]^{\text {! }}$ is a factor of $\left[\begin{array}{c}\mathrm{k}_{i} ; 0 \\ t_{i}\end{array}\right]$ for every $i$. Thus, 4.3 implies (1). If $|\mathbf{t}|=r$, (1) implies that $\left(\mathrm{k}_{i}-v^{t_{i}}\right) \mathrm{k}_{\mathbf{t}}=0$, proving the first (and hence the last) assertion in (2).

The subalgebra $\mathbf{S}_{r}^{0}$ of $\mathbf{S}_{r}$ generated by the $\mathrm{k}_{i}$ is a quotient of $\mathbf{U}^{0}$. Let

$$
\Lambda(n, r)=\left\{\mathbf{t}\left|\mathbf{t} \in \mathbb{N}^{n},\right| \mathbf{t} \mid=r\right\}
$$

be the set of compositions of $r$, and let $\unrhd$ be the dominance partial ordering on $\Lambda(n, r): \lambda \unrhd \mu \Longleftrightarrow \sum_{i=1}^{j} \lambda_{i} \geqslant \sum_{i=1}^{j} \mu_{i}, \forall j$. We have the following result (cf. [8] $7.4(\mathrm{~b})])$. 
Corollary 4.6. The algebra $\mathbf{S}_{r}^{0}$ is a commutative semisimple algebra over $\mathbb{Q}(v)$. The set $\left\{\mathrm{k}_{\lambda}\right\}_{\lambda \in \Lambda(n, r)}$ is a complete set of primitive orthogonal idempotents (hence a basis) for $\mathbf{S}_{r}^{0}$. In particular, the identity element $1 \in \mathbf{S}_{r}$ has the form $1=$ $\sum_{\lambda \in \Lambda(n, r)} \mathrm{k}_{\lambda}$.

Proof. Let $\lambda^{+}$be the partition obtained by permuting the components of $\lambda \in$ $\Lambda(n, r)$. By 4.4 2$), \mathrm{k}_{\lambda}$ acts on $L_{v}\left(\lambda^{+}\right)_{\lambda} \neq 0$ as an identity operator. Hence, $\mathrm{k}_{\lambda} \neq 0$. By 4.5 (2), $\mathrm{k}_{\lambda} \mathrm{k}_{\mu}=\delta_{\lambda, \mu} \mathrm{k}_{\lambda}$ for all $\lambda, \mu \in \Lambda(n, r)$; so the $\mathrm{k}_{\lambda}$ are nonzero orthogonal idempotents. The relations given in 4.1 imply that the $\# \Lambda(n, r)$ monomials $\mathrm{k}_{1}^{j_{1}} \cdots \mathrm{k}_{n-1}^{j_{n-1}}$ in the $\mathrm{k}_{i}$ of total degree at most $r$ span $\mathbf{S}_{r}^{0}$. Thus, by dimension considerations, the linearly independent elements $\mathrm{k}_{\lambda}, \lambda \in \Lambda(n, r)$, must be a basis for $\mathbf{S}_{r}^{0}$. The corollary now follows.

A dimension comparison gives rise to other bases for $\mathbf{S}_{r}^{0}$; e.g., part (1) of the corollary below follows from the proof above.

Corollary 4.7. (1) The elements $\mathrm{k}_{1}^{j_{1}} \cdots \mathrm{k}_{n-1}^{j_{n-1}}\left(j_{i} \in \mathbb{N}, j_{1}+\cdots+j_{n-1} \leqslant r\right)$ form a basis for $\mathbf{S}_{r}^{0}$.

(2) For any $\lambda \in \Lambda(n, r)$, let

$$
\mathrm{k}_{\lambda}^{\prime}:=\left[\begin{array}{c}
\mathrm{k}_{1} ; 0 \\
\lambda_{1}
\end{array}\right]\left[\begin{array}{c}
\mathrm{k}_{2} ; 0 \\
\lambda_{2}
\end{array}\right] \ldots\left[\begin{array}{c}
\mathrm{k}_{n-1} ; 0 \\
\lambda_{n-1}
\end{array}\right] .
$$

Then the set $\left\{\mathbf{k}_{\lambda}^{\prime}\right\}_{\lambda \in \Lambda(n, r)}$ forms a basis for $\mathbf{S}_{r}^{0}$.

Proof. We prove (2). For $\lambda, \mu \in \Lambda(n, r)$, write $\lambda \leqslant \mu \Longleftrightarrow \lambda_{i} \leqslant \mu_{i}$, for all $i=$ $1,2, \cdots, n-1$. Set $\lambda<\mu$ if also $\lambda_{i}<\mu_{i}$ for some $i$. By 4.5 $\mathrm{k}_{\mu}^{\prime} \mathrm{k}_{\lambda} \neq 0 \Longleftrightarrow \mu \leqslant \lambda$. So 4.6 implies

$$
\mathrm{k}_{\mu}^{\prime}=\sum_{\lambda \in \Lambda(n, r), \mu \leqslant \lambda} \mathrm{k}_{\mu}^{\prime} \mathrm{k}_{\lambda}=\mathrm{k}_{\mu}+\sum_{\lambda \in \Lambda(n, r), \mu<\lambda} \prod_{i=1}^{n-1}\left[\begin{array}{l}
\lambda_{i} \\
\mu_{i}
\end{array}\right] \mathrm{k}_{\lambda} .
$$

Now the assertion (2) follows easily.

For $A \in \Xi$ and $1 \leqslant i \leqslant n$, define

$$
\sigma_{i}(A)=a_{i, i}+\sum_{1 \leqslant j<i}\left(a_{i, j}+a_{j, i}\right), \quad \sigma_{i}^{\prime}(A)=a_{i, i}+\sum_{i<j \leqslant n}\left(a_{i, j}+a_{j, i}\right) .
$$

Then

$$
\#\left\{(\lambda, A) \mid \lambda \in \Lambda(n, r), A \in \Xi^{ \pm}, \lambda_{i} \geqslant \sigma_{i}(A) \forall i\right\}=\left(\begin{array}{c}
r+n^{2}-1 \\
r
\end{array}\right) .
$$

To see this, put $a_{i, i}=\lambda_{i}-\sigma_{i}(A)$. Then the cardinality above is the number of matrices $\left(a_{i, j}\right) \in \Xi$ such that $\sum_{i, j} a_{i, j}=r$. The identity holds if $\sigma_{i}$ is replaced by $\sigma_{i}^{\prime}$.

The next result was observed in [7 4.6] and generalized in [8, 7.9]. For $1 \leqslant i \leqslant$ $n-1$, let

$$
\alpha_{i}=\left(0, \cdots, 0,{ }_{i}^{1},-1,0, \cdots, 0\right) .
$$

Lemma 4.8. Let $\lambda \in \Lambda(n, r)$.

(1) If $\lambda_{i+1} \geqslant 1$, then $\mathrm{e}_{i} \mathrm{k}_{\lambda}=\mathrm{k}_{\lambda+\alpha_{i}} \mathrm{e}_{i}$.

(2) If $\lambda_{i} \geqslant 1$, then $\mathrm{f}_{i} \mathrm{k}_{\lambda}=\mathrm{k}_{\lambda-\alpha_{i}} \mathrm{f}_{i}$. 
Proof. Formula (2) results by applying the anti-automorphism $\tau$ given in 4.2 (1) to (1). We prove (1). By 2.3(5), we have

$$
\mathrm{e}_{i} \mathrm{k}_{\lambda}=\prod_{j \neq i, i+1}\left[\begin{array}{c}
\mathrm{k}_{j} ; 0 \\
\lambda_{j}
\end{array}\right]\left[\begin{array}{c}
\mathrm{k}_{i} ;-1 \\
\lambda_{i}
\end{array}\right]\left[\begin{array}{c}
\mathrm{k}_{i+1} ; 1 \\
\lambda_{i+1}
\end{array}\right] \mathrm{e}_{i} .
$$

Multiplying on the left by $\left[\begin{array}{c}\mathrm{k}_{i} ; 0 \\ 1\end{array}\right]$ and applying [2.3(1),(5) and 4.5)(2) gives

$$
\left[\begin{array}{c}
\lambda_{i}+1 \\
1
\end{array}\right] \mathrm{e}_{i} \mathrm{k}_{\lambda}=\left[\begin{array}{c}
\lambda_{i}+1 \\
1
\end{array}\right] \prod_{j \neq i, i+1}\left[\begin{array}{c}
\mathrm{k}_{j} ; 0 \\
\lambda_{j}
\end{array}\right]\left[\begin{array}{c}
\mathrm{k}_{i} ; 0 \\
\lambda_{i}+1
\end{array}\right]\left[\begin{array}{c}
\mathrm{k}_{i+1} ; 1 \\
\lambda_{i+1}
\end{array}\right] \mathrm{e}_{i}
$$

By 2.3(4) and 4.5, (1) follows after cancelling $\left[\begin{array}{c}\lambda_{i}+1 \\ 1\end{array}\right]$.

Recall the sequence $r o(A)$ (resp., $c o(A)$ ) of row (resp., column) sums of $A$ defined in $\S 2$.

Corollary 4.9. Let $A \in \Xi^{ \pm}$and $\lambda \in \Lambda(n, r)$.

(1) If $\lambda_{i} \geqslant \sigma_{i}\left(A^{+}\right), 1 \leqslant i \leqslant n$, then $\mathrm{e}^{\left(A^{+}\right)} \mathrm{k}_{\lambda}=\mathrm{k}_{\lambda^{\prime}} \mathrm{e}^{\left(A^{+}\right)}$, where $\lambda^{\prime}=\lambda-$ $\operatorname{co}\left(A^{+}\right)+\operatorname{ro}\left(A^{+}\right)$.

(2) If $\lambda_{i} \geqslant \sigma_{i}\left(A^{-}\right), 1 \leqslant i \leqslant n$, then $\mathrm{k}_{\lambda} \mathrm{f}^{\left(A^{-}\right)}=\mathrm{f}^{\left(A^{-}\right)} \mathrm{k}_{\lambda^{\prime \prime}}$, where $\lambda^{\prime \prime}=\lambda+$ $\operatorname{co}\left(A^{-}\right)-\operatorname{ro}\left(A^{-}\right)$.

In these cases, we have $\lambda^{\prime} \unrhd \lambda$ and $\lambda^{\prime \prime} \unrhd \lambda$.

Proof. If $i<j$ and $a \leqslant \lambda_{l}$ for all $l=i+1, \cdots, j$, by 4.8 ,

$$
\left(\mathrm{e}_{i}^{a} \cdots \mathrm{e}_{j-1}^{a}\right) \mathrm{k}_{\lambda}=\mathrm{k}_{\mu}\left(\mathrm{e}_{i}^{a} \cdots \mathrm{e}_{j-1}^{a}\right)
$$

where $\mu=\lambda+a\left(\alpha_{i}+\cdots+\alpha_{j-1}\right)$. Now assume that $\lambda_{i} \geqslant \sigma_{i}\left(A^{+}\right)$for all $i$. Since $\mathrm{e}^{\left(A^{+}\right)}$is a product of terms $\mathrm{e}_{i}^{\left(a_{i, j}\right)} \cdots \mathrm{e}_{j-1}^{\left(a_{i, j}\right)}$, we obtain that $\mathrm{e}^{\left(A^{+}\right)} \mathrm{k}_{\lambda}=\mathrm{k}_{\lambda^{\prime}} \mathrm{e}^{\left(A^{+}\right)}$for $\lambda^{\prime}=\lambda+\sum_{i<j} a_{i, j}\left(\alpha_{i}+\cdots+\alpha_{j-1}\right)$, that is,

$$
\begin{aligned}
\lambda_{1}^{\prime} & =\lambda_{1}+a_{1,2}+\cdots+a_{1, n}, \\
\lambda_{2}^{\prime} & =\lambda_{2}-a_{1,2}+a_{2,3}+\cdots+a_{2, n}, \\
\lambda_{3}^{\prime} & =\lambda_{3}-\left(a_{1,3}+a_{2,3}\right)+a_{3,4}+\cdots+a_{3, n}, \\
& \cdots \\
\lambda_{n-1}^{\prime} & =\lambda_{n-1}-\left(a_{1, n-1}+\cdots+a_{n-2, n-1}\right)+a_{n-1, n}, \\
\lambda_{n}^{\prime} & =\lambda_{n}-\left(a_{1, n}+\cdots+a_{n-1, n}\right),
\end{aligned}
$$

yielding the required formula. Applying $\tau$ in 4.2 to the identity in (1) gives that in (2). The last assertion follows easily from the definition.

For part (1) of the following result, see also [8, 7.9].

Lemma 4.10. Let $\lambda \in \Lambda(n, r)$.

(1) If $\lambda_{i+1}=0$ for some $1 \leqslant i \leqslant n-1$, then $\mathrm{e}_{i} \mathrm{k}_{\lambda}=\mathrm{k}_{\lambda} \mathrm{f}_{i}=0$.

(2) More generally, if $A \in \Xi^{+}$(resp., $A \in \Xi^{-}$) and $\lambda_{i}<\sigma_{i}(A)$ for some $i$, then $\mathrm{e}^{(A)} \mathrm{k}_{\lambda}=0$ (resp., $\left.\mathrm{k}_{\lambda} \mathrm{f}^{(A)}=0\right)$.

Proof. It suffices to prove (1) for $\mathrm{e}_{i}$ and (2) for $A \in \Xi^{+}$; the others can be obtained by applying the anti-automorphism $\tau$, since $\tau\left(\mathrm{e}^{(A)}\right)=\mathrm{f}^{\left(A^{T}\right)}$ if $A^{T}$ is the transpose of $A \in \Xi^{+}$. 
To prove (1), assume $\lambda_{i+1}=0$. By 4.1 and 4.5(2), $\mathrm{k}_{i+1} \mathrm{e}_{i} \mathrm{k}_{\lambda}=v^{-1} \mathrm{e}_{i} \mathrm{k}_{\lambda}$. Also, 4.1 and 4.6 imply that $\mathrm{e}_{i} \mathrm{k}_{\lambda}$ is a $\mathbb{Q}(v)$-linear combination of terms $\mathrm{k}_{\mu} \mathrm{e}_{i}, \mu \in \Lambda(n, r)$. Since $\mathrm{k}_{i+1} \mathrm{k}_{\mu} \mathrm{e}_{i}=v^{\mu_{i+1}} \mathrm{k}_{\mu} \mathrm{e}_{i}$ and $\mu_{i+1} \geqslant 0$, it follows that $\mathrm{e}_{i} \mathrm{k}_{\lambda}=0$.

To prove (2), let $i$ be minimal with $\lambda_{i}<\sigma_{i}(A)$. Then $i>1$, since $\sigma_{1}(A)=0$. Suppose $a_{1, i}+\cdots+a_{i^{\prime}-1, i} \leqslant \lambda_{i}<a_{1, i}+\cdots+a_{i^{\prime}, i}$ for some $1 \leqslant i^{\prime} \leqslant i-1$. Write $\left[\begin{array}{c}a_{i^{\prime}, i} \\ x\end{array}\right] \mathrm{e}^{(A)}=\mathrm{m}_{1} \mathrm{~m}_{2}$, where

$$
\mathrm{m}_{1}=\mathrm{e}_{n-1}^{\left(a_{n-1, n}\right)} \cdots \mathrm{e}_{i-1}^{\left(a_{i^{\prime}, i}-x\right)}, \quad \mathrm{m}_{2}=\mathrm{e}_{i-1}^{(x)} \mathrm{e}_{i^{\prime}-1}^{\left(a_{i^{\prime}-1, i}\right)} \mathrm{e}_{i^{\prime}}^{\left(a_{i^{\prime}-1, i}\right)} \cdots \mathrm{e}_{i-1}^{\left(a_{i^{\prime}-1, i}\right)} \cdots \mathrm{e}_{1}^{\left(a_{1,2}\right)}
$$

and $x=\lambda_{i}-\left(a_{1, i}+\cdots+a_{i^{\prime}-1, i}\right)$. By 4.9 and 4.8 $\left[\begin{array}{c}a_{i^{\prime}, i} \\ x\end{array}\right] \mathrm{e}^{(A)} \mathrm{k}_{\lambda}=\mathrm{m}_{1} \mathrm{k}_{\mu} \mathrm{m}_{2}$, where $\mu=\left(\mu_{1}, \cdots, \mu_{n}\right) \in \Lambda(n, r)$ with $\mu_{i}=\left(\lambda_{i}-a_{1, i}-\cdots-a_{i^{\prime}-1, i}\right)-x=0$. Now, since $a_{i^{\prime}, i}-x>0$, we have $\mathrm{m}_{1} \mathrm{k}_{\mu}=\mathrm{m}_{1}^{\prime} \mathrm{e}_{i-1} \mathrm{k}_{\mu}=0$ by part $(1)$, and $\mathrm{e}^{(A)} \mathrm{k}_{\lambda}=0$.

Let $\mathbf{S}_{r}^{+}$(resp., $\mathbf{S}_{r}^{-}$) be the subalgebra of $\mathbf{S}_{r}$ generated by the $\mathrm{e}_{i}$ (resp., $\mathbf{f}_{i}$ ). Using PBW bases, [14, 2.5] gives a version of the following result; see also $\S 6$ below.

Corollary 4.11. The algebra $\mathbf{S}_{r}^{+}$(resp., $\mathbf{S}_{r}^{-}$) is spanned by the elements

$$
\left\{\mathrm{e}^{(A)}: A \in \Xi^{+}, \sigma(A) \leqslant r\right\} \quad\left(\text { resp. },\left\{\mathrm{f}^{(A)}: A \in \Xi^{-}, \sigma(A) \leqslant r\right\}\right) .
$$

Proof. If $\sigma\left(A^{+}\right)=\sum_{i} \sigma_{i}\left(A^{+}\right)>r$, then $\mathrm{e}^{(A)}=\sum_{\lambda \in \Lambda(n, r)} \mathrm{e}^{(A)} \mathrm{k}_{\lambda}=0$ by 4.6 and 4.10(2). The result follows since $\mathbf{S}_{r}^{+}$is spanned by all $\mathrm{e}^{(A)}$ with $A \in \Xi^{+}, \sigma(A) \leqslant r$, by 3.3 .

For $A=\left(a_{i, j}\right) \in \Xi$, let $b_{i, j}=a_{n-j+1, n-i+1}$. So ${ }^{T} A:=\left(b_{i, j}\right)$ is the matrix obtained by transposing $A$ along its skew-diagonal. Thus, $\sigma_{i}^{\prime}\left({ }^{T} A\right)=\sigma_{n-i+1}(A)$. The following result is an application of the anti-automorphism $\gamma$ in $4.2(2)$. Part (1) is a special case of [8] 7.9].

Corollary 4.12. Let $\lambda \in \Lambda(n, r)$.

(1) If $\lambda_{i}=0$ for some $i$ with $1 \leqslant i \leqslant n-1$, then $\mathrm{k}_{\lambda} \mathbf{e}_{i}=\mathbf{f}_{i} \mathrm{k}_{\lambda}=0$.

(2) More generally, if $A \in \Xi^{+}$(resp., $A \in \Xi^{-}$) and $\lambda_{i}<\sigma_{i}^{\prime}(A)$ for some $i$, then $\mathrm{k}_{\lambda} \gamma\left(\mathrm{e}^{\left({ }^{T} A\right)}\right)=0$ (resp., $\left.\gamma\left(\mathrm{f}^{\left({ }^{T} A\right)}\right) \mathrm{k}_{\lambda}=0\right)$.

Proof. Define $\lambda^{\mathrm{op}}=\left(\lambda_{1}^{\mathrm{op}}, \cdots, \lambda_{n}^{\mathrm{op}}\right)$ by reversing the components of $\lambda=\left(\lambda_{1}, \cdots, \lambda_{n}\right)$ (i.e., $\left.\lambda_{i}^{\mathrm{op}}=\lambda_{n-i+1}\right)$. Then, $\gamma\left(\mathrm{k}_{\lambda}\right)=\mathrm{k}_{\lambda^{\mathrm{op}}}$, and (1) follows easily from4.10(1). Since $\lambda_{i}<\sigma_{i}^{\prime}(A)$ means $\lambda_{n-i+1}^{\mathrm{op}}<\sigma_{n-i+1}\left({ }^{T} A\right)$, by 4.10 $(2), \mathrm{e}^{\left({ }^{T} A\right)} \mathrm{k}_{\lambda_{\mathrm{op}}}=0=\mathrm{k}_{\lambda_{\mathrm{op}} \mathrm{f}}{ }^{\left({ }^{T} A\right)}$. Now apply $\gamma$.

The elements $\gamma\left(\mathrm{e}^{\left({ }^{T} A^{+}\right)}\right)$and $\gamma\left(\mathrm{f}^{\left({ }^{T} A^{-}\right)}\right)$can be explicitly described as follows: for the $j$ th row (reading to the left) $a_{j, n}, \cdots, a_{j, j+1}(1 \leqslant j \leqslant n-1)$ of $A^{+}$, put

$$
\mathrm{n}_{j}=\left(\mathrm{e}_{j}^{\left(a_{j, n}\right)} \mathrm{e}_{j+1}^{\left(a_{j, n}\right)} \cdots \mathrm{e}_{n-1}^{\left(a_{j, n}\right)}\right) \cdots\left(\mathrm{e}_{j}^{\left(a_{j, j+2}\right)} \mathrm{e}_{j+1}^{\left(a_{j, j+2}\right)}\right) \mathrm{e}_{j}^{\left(a_{j, j+1}\right)} .
$$

Similarly, for the $j$-th column (reading downwards) $a_{j+1, j}, \cdots, a_{n, j}$ of $A^{-}$, put

$$
\mathbf{n}_{j}^{\prime}=\mathbf{f}_{j}^{\left(a_{j+1, j}\right)}\left(\mathbf{f}_{j+1}^{\left(a_{j+2, j}\right)} \mathbf{f}_{j}^{\left(a_{j+2, j}\right)}\right) \cdots\left(\mathbf{f}_{n-1}^{\left(a_{n, j}\right)} \mathbf{f}_{n-2}^{\left(a_{n, j}\right)} \cdots \mathbf{f}_{j}^{\left(a_{n, j}\right)}\right) .
$$

Then $\gamma\left(\mathrm{e}^{\left({ }^{T} A^{+}\right)}\right)=\mathrm{n}_{n-1} \mathrm{n}_{n-2} \cdots \mathrm{n}_{1}$ and $\gamma\left(\mathrm{f}^{\left({ }^{T} A^{-}\right)}\right)=\mathrm{n}_{1}^{\prime} \mathrm{n}_{2}^{\prime} \cdots \mathrm{n}_{n-1}^{\prime}$.

The following is the "little" version of 3.4 , from which it follows.

Lemma 4.13. Let $A=\left(a_{i, j}\right) \in \Xi^{+}$and let $\mathrm{m} \in \mathbf{S}_{r}$ be any monomial in the $\mathbf{e}_{i}$. Then the product $\mathrm{e}^{(A)}$ is a linear combination of $\mathrm{e}^{(B)}$ with $B \in \Xi^{+}$(and hence, of $\mathrm{e}^{(B)} \mathrm{k}_{\lambda}$ with $\left.\lambda \in \Lambda(n, r), B \in \Xi^{+}\right)$, and $\operatorname{deg}(B) \leqslant \operatorname{deg}(\mathrm{m})+\operatorname{deg}(A)$. In particular, 
$\mathrm{m}$ itself can be written as a linear combination of $\mathrm{e}^{(B)} \mathrm{k}_{\lambda}$ with $\lambda \in \Lambda(n, r), B \in \Xi^{+}$ and $\operatorname{deg}(B) \leqslant \operatorname{deg}(\mathrm{m})$. A similar result holds for the negative part of the algebra.

For any $A \in \Xi^{ \pm}$and $\lambda \in \Lambda(n, r)$, let

$$
\mathrm{m}^{(A, \lambda)}=\mathrm{e}^{\left(A^{+}\right)} \mathrm{k}_{\lambda} \mathrm{f}^{\left(A^{-}\right)} .
$$

By 3.3 , 4.6 and 4.10 (2), $\mathbf{S}_{r}$ is spanned by all such $\mathrm{m}^{(A, \lambda)}$ with $\lambda \in \Lambda(n, r)$, and $A \in \Xi^{ \pm}$satisfying $\sigma\left(A^{+}\right) \leqslant r$ and $\sigma\left(A^{-}\right) \leqslant r$.

Theorem 4.14. For $A=\left(a_{i, j}\right) \in \Xi_{r}$, let

$$
\mathrm{m}^{(A)}=\prod_{1 \leqslant i \leqslant h<j \leqslant n} \mathrm{e}_{h}^{\left(a_{i, j}\right)} \prod_{i=1}^{n}\left[\begin{array}{c}
\mathrm{k}_{i} ; 0 \\
\lambda_{i}
\end{array}\right] \prod_{1 \leqslant j \leqslant h<i \leqslant n} \mathrm{f}_{h}^{\left(a_{i, j}\right)}=\mathrm{e}^{\left(A^{+}\right)} \mathrm{k}_{\lambda} \mathrm{f}^{\left(A^{-}\right)},
$$

where $\lambda=\lambda(A)=\left(\sigma_{1}(A), \cdots, \sigma_{n}(A)\right)$. The set $\mathfrak{M}=\left\{\mathrm{m}^{(A)}\right\}_{A \in \Xi_{r}}$ is a spanning set for $\mathbf{S}_{r}$.

Proof. Fix $B \in \Xi^{ \pm}$satisfying $\sigma\left(B^{+}\right) \leqslant r$ and $\sigma\left(B^{-}\right) \leqslant r$. Let $\lambda \in \Lambda(n, r)$. If $\lambda_{i} \geq \sigma_{i}(B)$ for all $i$, then there is a unique $A \in \Xi_{r}$ such that $\mathrm{m}^{(A)}=\mathrm{m}^{(B, \lambda)}$. Therefore, to prove the theorem, we must show that if $\lambda_{i}<\sigma_{i}(B)$ for some $i$, then $\mathrm{m}^{(B, \lambda)}$ lies in the span of $\mathfrak{M}$. We proceed by induction on $\operatorname{deg}(B)$; cf. 3.1.1). The result follows from 4.10 if $\operatorname{deg}(B)=1$. Assume now that $\operatorname{deg}(B)>1$, and suppose $i$ is minimal with $\lambda_{i}<\sigma_{i}(B)$. Let $B_{i}$ be the submatrix of $B$ consisting of the first $i$ rows and columns, and write $\mathrm{e}^{\left(B^{+}\right)}=\mathrm{m}_{1} \mathrm{e}^{\left(B_{i}^{+}\right)}$and $\mathrm{f}^{\left(B^{-}\right)}=\mathrm{f}^{\left(B_{i}^{-}\right)} \mathrm{m}_{1}^{\prime}$. Then

$$
\mathrm{m}^{(B, \lambda)}=\mathrm{m}_{1} \mathrm{e}^{\left(B_{i}^{+}\right)} \mathrm{k}_{\lambda} \mathrm{f}^{\left(B_{i}^{-}\right)} \mathrm{m}_{1}^{\prime} .
$$

By $4.10(2)$, we can assume $\lambda_{i} \geqslant \sigma_{i}\left(B^{+}\right)$(and so $\lambda_{j} \geqslant \sigma_{j}\left(B^{+}\right)$for all $1 \leqslant j \leqslant i$ by the minimality assumption on $i$ ). Now 4.9 (1) implies

$$
\mathrm{m}^{(B, \lambda)}=\mathrm{m}_{1}\left(\mathrm{e}^{\left(B_{i}^{+}\right)} \mathrm{k}_{\lambda}\right) \mathrm{f}^{\left(B_{i}^{-}\right)} \mathrm{m}_{1}^{\prime}=\mathrm{m}_{1} \mathrm{k}_{\lambda^{\prime}} \mathrm{e}^{\left(B_{i}^{+}\right)} \mathrm{f}^{\left(B_{i}^{-}\right)} \mathrm{m}_{1}^{\prime},
$$

where $\lambda^{\prime}=\left(\lambda_{1}^{\prime}, \cdots, \lambda_{n}^{\prime}\right)$ with $\lambda_{i}^{\prime}=\lambda_{i}-\left(a_{1, i}+\cdots+a_{i-1, i}\right)=\lambda_{i}-\sigma_{i}\left(B_{i}^{+}\right) \geqslant 0$; cf. the last equation in (4.9.1). By remarks after 2.3,

$$
\mathrm{e}^{\left(B_{i}^{+}\right)} \mathrm{f}^{\left(B_{i}^{-}\right)}=\mathrm{f}^{\left(B_{i}^{-}\right)} \mathrm{e}^{\left(B_{i}^{+}\right)}+f
$$

where $f$ is a linear combination of monomials $\mathrm{m}_{j}^{\mathrm{e}} \mathrm{h}_{j} \mathrm{~m}_{j}^{\mathrm{f}}$ with $\mathrm{h}_{j} \in \mathbf{S}_{r}^{0}$ and $\operatorname{deg}\left(\mathrm{m}_{j}^{\mathrm{e}} \mathrm{m}_{j}^{\mathrm{f}}\right)<$ $\operatorname{deg}\left(B_{i}\right)$. Here, $\mathrm{m}_{j}^{\mathrm{e}}$ (resp., $\mathrm{m}_{j}^{\mathrm{f}}$ ) denotes a monomial in the $\mathrm{e}_{i}$ (resp., $\mathrm{f}_{i}$ ). Thus, $\operatorname{deg}\left(\mathrm{m}_{1} \mathrm{~m}_{j}^{\mathrm{e}} \mathrm{m}_{j}^{\mathrm{f}} \mathrm{m}_{1}^{\prime}\right)<\operatorname{deg}(B)$. Since $\lambda_{i}^{\prime}<\sigma_{i}\left(B_{i}^{-}\right), \mathrm{m}_{1} \mathrm{k}_{\lambda^{\prime}} \mathrm{f}^{\left(B_{i}^{-}\right)} \mathrm{e}^{\left(B_{i}^{+}\right)}=0$ by 4.10, By 4.13, $\mathrm{m}_{1} \mathrm{~m}_{j}^{\mathrm{e}}$ (resp., $\mathrm{m}_{j}^{\mathrm{f}} \mathrm{m}_{1}^{\prime}$ ) is a linear combination of $\mathrm{e}^{(C)} \mathrm{k}_{\lambda^{\prime \prime}}, C \in \Xi^{+}$(resp., $\mathrm{f}^{\left(C^{\prime}\right)} \mathrm{k}_{\lambda^{\prime \prime}}$, $C^{\prime} \in \Xi^{-}$) with $\operatorname{deg}(C) \leqslant \operatorname{deg}\left(\mathrm{m}_{1} \mathrm{~m}_{j}^{\mathrm{e}}\right)$ (resp., $\operatorname{deg}\left(C^{\prime}\right) \leqslant \operatorname{deg}\left(\mathrm{m}_{j}^{\mathrm{f}} \mathrm{m}_{1}^{\prime}\right)$ ). Thus, each $\mathrm{m}_{1} \mathrm{k}_{\lambda^{\prime}} \mathrm{m}_{j}^{\mathrm{e}} \mathrm{h}_{j} \mathrm{~m}_{j}^{\mathrm{f}} \mathrm{m}_{1}^{\prime}\left(=\mathrm{m}_{1} \mathrm{~m}_{j}^{\mathrm{e}} \mathrm{k}_{\lambda^{\prime \prime}} \mathrm{h}_{j} \mathrm{~m}_{j}^{\mathrm{f}} \mathrm{m}_{1}^{\prime}\right)$ is a linear combination of $\mathrm{m}^{\left(B^{\prime}, \mu\right)}$ with $\operatorname{deg}\left(B^{\prime}\right)<$ $\operatorname{deg}(B)$, since $\operatorname{deg}\left(\mathrm{m}_{1} \mathrm{~m}_{j}^{\mathrm{e}} \mathrm{m}_{j}^{\mathrm{f}} \mathrm{m}_{1}^{\prime}\right)<\operatorname{deg}(B)$. By induction, $\mathrm{m}^{(B, \lambda)}$ is in the span of $\mathfrak{M}$.

Note that all elements in $\mathfrak{M}$ are fixed under the involution ${ }^{-}$defined in $4.2(3)$. 


\section{The isomorphism Between $\mathbf{S}_{r}$ AND $\mathbf{U}_{r}$}

Recall from $3.1(3)$ the surjective algebra homomorphism $\zeta_{r}: \mathbf{U} \rightarrow \mathbf{U}_{r}$. Since

$$
\zeta_{r}\left(K_{i}\right)=\zeta_{r}\left(0\left(\mathbf{e}_{i}\right)\right)=0\left(\mathbf{e}_{i}, r\right)=\sum_{\substack{D \in \Xi_{r}^{0} \\ D=\operatorname{diag}\left(d_{1}, \cdots, d_{n}\right)}} v^{d_{i}}[D]
$$

where $\mathbf{e}_{i}=\left(0, \cdots, 0,{ }_{i}^{1}, 0, \cdots, 0\right)$, and since $[D]\left[D^{\prime}\right]=\delta_{D, D^{\prime}}[D]$, we obtain the following.

Lemma 5.1. We have $\zeta_{r}\left(K_{1} K_{2} \cdots K_{n}\right)=v^{r} 1$.

For any $i, j, 1 \leqslant i \leqslant n, 0 \leqslant j \leqslant r$, let

$$
\mathfrak{d}_{i}(j)=\sum_{D \in \Xi_{r}^{0}, d_{i}=j}[D]
$$

so $\zeta_{r}\left(K_{i}\right)=\sum_{j=0}^{r} v^{j} \mathfrak{d}_{i}(j)$.

Lemma 5.2. For any $\mathbf{t} \in \mathbb{N}^{n}$, let

$$
K(\mathbf{t})=\left[K_{1} ; t_{1}\right]^{!}\left[K_{2} ; t_{2}\right]^{!} \cdots\left[K_{n} ; t_{n}\right]^{!} .
$$

Then $\zeta_{r}(K(\mathbf{t}))=0$ whenever $|\mathbf{t}|>r$.

Proof. Since $\zeta_{r}\left(K_{i}-v^{d}\right)=\sum_{j_{i}=0}^{r}\left(v^{j_{i}}-v^{d}\right) \mathfrak{d}_{i}\left(j_{i}\right)$, we have

$\zeta_{r}\left(\left[K_{i} ; t_{i}\right]^{!}\right)=\sum_{j_{i}=0}^{r}\left(v^{j_{i}}-1\right)\left(v^{j_{i}}-v\right) \cdots\left(v^{j_{i}}-v^{t_{i}-1}\right) \mathfrak{d}_{i}\left(j_{i}\right)=\sum_{j_{i}=t_{i}}^{r} \prod_{l=0}^{t_{i}-1}\left(v^{j_{i}}-v^{l}\right) \mathfrak{d}_{i}\left(j_{i}\right)$.

Thus, if $|\mathbf{t}|>r$, the fact that $j_{1}+\cdots+j_{n} \geqslant t_{1}+\cdots+t_{n}>r$ implies

$$
\zeta_{r}(K(\mathbf{t}))=\sum_{j_{1} \geqslant t_{1}, \cdots, j_{n} \geqslant t_{n}}\left(\prod_{i=1}^{n} \prod_{l=0}^{t_{i}-1}\left(v^{j_{i}}-v^{l}\right)\right) \mathfrak{d}_{1}\left(j_{1}\right) \cdots \mathfrak{d}_{n}\left(j_{n}\right)=0,
$$

since $\mathfrak{d}_{1}\left(j_{1}\right) \mathfrak{d}_{2}\left(j_{2}\right) \cdots \mathfrak{d}_{n}\left(j_{n}\right)=0$, whenever $j_{1}+j_{2}+\cdots+j_{n}>r$.

The following has already been obtained in [14, 2.10] using [9, (3.4.a)].

Corollary 5.3. For any $\mathbf{t} \in \mathbb{N}^{n}$, let $K_{\mathbf{t}}=\prod_{i=1}^{n}\left[\begin{array}{c}K_{i} ; 0 \\ t_{i}\end{array}\right]$. Then

$$
\zeta_{r}\left(K_{\mathbf{t}}\right)= \begin{cases}0, & \text { if }|\mathbf{t}|>r \\ {\left[\operatorname{diag}\left(t_{1}, \cdots, t_{n}\right)\right],} & \text { if }|\mathbf{t}|=r .\end{cases}
$$

Proof. The case for $|\mathbf{t}|>r$ follows from 5.2, If $|\mathbf{t}|=r$, 9, (3.4.a)] implies that

$$
\zeta_{r}\left[\begin{array}{c}
K_{i} ; 0 \\
t_{i}
\end{array}\right]=\sum_{j_{i}=0}^{r}\left[\begin{array}{c}
j_{i} \\
t_{i}
\end{array}\right] \mathfrak{d}_{i}\left(j_{i}\right)=\sum_{j_{i}=t_{i}}^{r}\left[\begin{array}{c}
j_{i} \\
t_{i}
\end{array}\right] \mathfrak{d}_{i}\left(j_{i}\right)
$$

since $\left[\begin{array}{l}j \\ t\end{array}\right]=0$ for $0 \leqslant j<t$. Thus,

$$
\zeta_{r}\left(\prod_{i=1}^{n}\left[\begin{array}{c}
K_{i} ; 0 \\
t_{i}
\end{array}\right]\right)=\sum_{j_{1} \geqslant t_{1}, \cdots, j_{n} \geqslant t_{n}} \prod_{i=1}^{n}\left[\begin{array}{l}
j_{i} \\
t_{i}
\end{array}\right] \mathfrak{d}_{1}\left(j_{1}\right) \cdots \mathfrak{d}_{n}\left(j_{n}\right) .
$$

If $\mathfrak{d}_{1}\left(j_{1}\right) \cdots \mathfrak{d}_{n}\left(j_{n}\right) \neq 0$ here, then $j_{i}=t_{i}, \forall i$. Since $\mathfrak{d}_{1}\left(t_{1}\right) \mathfrak{d}_{2}\left(t_{2}\right) \cdots \mathfrak{d}_{n}\left(t_{n}\right)=$ $\left[\operatorname{diag}\left(t_{1}, \cdots, t_{n}\right)\right]$, the result follows. 
Note that we actually have $\mathfrak{d}_{1}\left(j_{1}\right) \mathfrak{d}_{2}\left(j_{2}\right) \cdots \mathfrak{d}_{n-1}\left(j_{n-1}\right)=\left[\operatorname{diag}\left(j_{1}, \cdots, j_{n}\right)\right]$, where $j_{n}=r-\left(j_{1}+j_{2}+\cdots+j_{n-1}\right)$. Thus, the proof above also shows that, for $\mathbf{t} \in \mathbb{N}^{n-1}$ with $|\mathbf{t}| \leqslant r$,

$$
\begin{aligned}
\zeta_{r}\left(\prod_{i=1}^{n-1}\left[\begin{array}{c}
K_{i} ; 0 \\
t_{i}
\end{array}\right]\right) & =\sum_{j_{1} \geqslant t_{1}, \cdots, j_{n-1} \geqslant t_{n-1}} \prod_{i=1}^{n-1}\left[\begin{array}{c}
j_{i} \\
t_{i}
\end{array}\right] \mathfrak{d}_{1}\left(j_{1}\right) \cdots \mathfrak{d}_{n-1}\left(j_{n-1}\right) \\
& =\sum_{\substack{\lambda \in \Lambda(n, r) \\
t_{j} \leqslant \lambda_{j}}} \prod_{i=1}^{n-1}\left[\begin{array}{c}
\lambda_{i} \\
t_{i}
\end{array}\right] \zeta_{r}\left(K_{\lambda}\right),
\end{aligned}
$$

yielding the $\mathbf{U}_{r}$ version of 4.7.1.

Theorem 5.4. The algebra homomorphism $\zeta_{r}: \mathbf{U} \rightarrow \mathbf{U}_{r}$ induces an isomorphism $\mathbf{S}_{r} \cong \mathbf{U}_{r}$. Moreover, the set $\mathfrak{M}$ given in 4.14 forms a basis for $\mathbf{S}_{r}$.

Proof. By [5.1 and 5.2 $\zeta_{r}$ induces a surjection $\bar{\zeta}_{r}: \mathbf{S}_{r} \rightarrow \mathbf{U}_{r}$. But 4.14 implies $\operatorname{dim} \mathbf{S}_{r} \leqslant \# \mathfrak{M}=\operatorname{dim} \mathbf{U}_{r} ;$ so $\bar{\zeta}_{r}$ is an isomorphism.

We call $\mathfrak{M}$ a monomial basis for $\mathbf{S}_{r}$. Using the isomorphism $\mathbf{S}_{r} \cong \mathbf{U}_{r}$, we identify the generators $\mathrm{e}_{i}$, etc. with $E_{i, i+1}(\mathbf{0}, r)$, etc. By 5.3,

$$
\mathrm{k}_{\lambda}=[\operatorname{diag}(\lambda)]:=\left[\operatorname{diag}\left(\lambda_{1}, \cdots, \lambda_{n}\right)\right], \quad \forall \lambda \in \Lambda(n, r) .
$$

Using [1 5.4], we can identify the elements $\left(m E_{h, h+1}\right)(\mathbf{0}, r)$ with $\mathrm{e}_{h}^{(m)}$, and the elements $\left(m E_{h+1, h}\right)(\mathbf{0}, r)$ with $\mathbf{f}_{h}^{(m)}$.

Recall the Bruhat ordering on $\Xi_{r}$ [1, 1.4]: Fix an algebraically closed field $k$, and let $V$ be an $r$-dimensional vector space over $k$. As shown in [1 1.1], the matrices $A \in \Xi_{r}$ correspond bijectively to $G L(V)$-orbits $\mathcal{O}_{A}$ of pairs of $n$-step filtrations of $V$. Then, given $A^{\prime}, A^{\prime \prime} \in \Xi_{r}, A^{\prime}<A^{\prime \prime}$ means $\mathcal{O}_{A^{\prime}} \subsetneq \overline{\mathcal{O}}_{A^{\prime \prime}}$. Here $\overline{\mathcal{O}}_{A^{\prime \prime}}$ denotes the Zariski closure of $\mathcal{O}_{A^{\prime \prime}}$. This partial ordering is independent of the algebraically closed field $k$.

We have the following identification of the monomial basis elements.

Theorem 5.5. For any $A \in \Xi_{r}, \mathrm{~m}^{(A)}$ is exactly the element defined in [1 3.9(a)]. In particular, in $\mathbf{U}_{r}$ we have

$$
\mathrm{m}^{(A)}=[A]+\sum_{B \in \Xi_{r}, B<A} f_{B, A}[B] \quad\left(f_{B, A} \in \mathcal{Z}\right) .
$$

Proof. By 4.14 and 4.9

$$
\mathrm{m}^{(A)}=\mathrm{e}^{\left(A^{+}\right)} \mathrm{k}_{\lambda} \mathrm{f}^{\left(A^{-}\right)}=\mathrm{k}_{\lambda^{\prime}} \mathrm{e}^{\left(A^{+}\right)} \mathrm{f}^{\left(A^{-}\right)}=\mathrm{e}^{\left(A^{+}\right)} \mathrm{f}^{\left(A^{-}\right)} \mathrm{k}_{\lambda^{\prime \prime}}
$$

with $\lambda^{\prime}=\lambda-\operatorname{co}\left(A^{+}\right)+\operatorname{ro}\left(A^{+}\right)$and $\lambda^{\prime \prime}=\lambda+\operatorname{co}\left(A^{-}\right)-\operatorname{ro}\left(A^{-}\right)$. Since $\lambda=\lambda(A)=$ $\left(\sigma_{1}(A), \cdots, \sigma_{n}(A)\right), \lambda^{\prime}=r o(A)$ and $\lambda^{\prime \prime}=c o(A)$. Recall that $\mathrm{e}^{\left(A^{+}\right)}\left(\right.$resp. $\left.\mathrm{f}^{\left(A^{-}\right)}\right)$is a product of $\mathrm{e}_{h}^{\left(a_{i, j}\right)}$ (resp. $\left.\mathrm{f}_{h}^{\left(a_{i, j}\right)}\right)$ for all $1 \leqslant i \leqslant h<j \leqslant n($ resp. $1 \leqslant j \leqslant h<i \leqslant n)$, which are ordered as in [1, 3.9(a)] (cf. $\S 3$ and footnote 6 above). If $\nu \in \Lambda(n, r)$ and $D=\operatorname{diag}(\nu)$ are such that $\mathrm{e}_{h}^{\left(a_{i, j}\right)} \mathrm{k}_{\nu} \neq 0$ then, by (3.0.3),

$$
\mathbf{e}_{h}^{\left(a_{i, j}\right)} \mathbf{k}_{\nu}=\left(a_{i, j} E_{h, h+1}\right)(\mathbf{0}, r)[D]=\left[a_{i, j} E_{h, h+1}+D^{\prime}\right]
$$

for a unique $D^{\prime} \in \Xi_{r}^{0}$ with $c o\left(a_{i, j} E_{h, h+1}+D^{\prime}\right)=\nu$. A similar statement holds for any $\mathbf{f}_{h}^{\left(a_{i, j}\right)} \mathrm{k}_{\nu}$. Thus, applying this and 4.9 (noting also that $\mathrm{k}_{\nu}^{2}=\mathrm{k}_{\nu}$ ) repeatedly 
from right to left beginning with $\mathrm{f}_{n-1}^{\left(a_{n, n-1}\right)} \mathrm{k}_{\lambda^{\prime \prime}}$, where $\mathrm{f}_{n-1}^{\left(a_{n, n-1}\right)}$ is the rightmost term of $\mathrm{f}^{\left(A^{-}\right)}$, we identify $\mathrm{m}^{(A)}$ with a product

$$
P=\prod_{1 \leqslant i \leqslant h<j \leqslant n}\left[D_{i, h, j}+a_{i, j} E_{h, h+1}\right] \times \prod_{1 \leqslant j \leqslant h<i \leqslant n}\left[D_{i, h, j}+a_{i, j} E_{h+1, h}\right] .
$$

Here the order of the factors follows that for $\mathrm{e}^{\left(A^{+}\right)}$and $\mathrm{f}^{\left(A^{-}\right)}$, and the diagonal matrices $D_{i, h, j} \in \Xi^{0}$ are inductively and uniquely determined by the conditions $c o\left(D_{n, n-1, n-1}+a_{n, n-1} E_{n, n-1}\right)=\lambda^{\prime \prime}=c o(A)(\mathrm{cf}$. 5.4.1) and $c o(X)=\operatorname{ro}(Y)$ if $[X]$, $[Y]$ are two adjacent terms in $P$. However, $P$ is identified in [1, 3.9(a)] as equaling an expression of the form given on the right-hand side of (5.5.1).

The following result will use the relation $\preccurlyeq$ on $\Xi$ defined above 3.2 , Observe that $\preccurlyeq$ does not involve diagonal entries: given $A, B \in \Xi$, put $A^{ \pm}=A^{+}+A^{-}$and $B^{ \pm}=B^{+}+B^{-}$. Then $A \preccurlyeq B \Longleftrightarrow A^{ \pm} \preccurlyeq B^{ \pm}$. In addition, 1, 3.6] states that for $A, B \in \Xi_{r}, A \leqslant B \Longrightarrow A \preccurlyeq B$ and $A<B \Longrightarrow A \prec B$. (For more results on these various poset structures, see [12, §5].)

Corollary 5.6. Suppose $\mathrm{m}^{(A, \lambda)} \neq 0$ for some $A \in \Xi^{ \pm}$and $\lambda \in \Lambda(n, r)$. If there exists $D \in \Xi^{0}$ such that $\operatorname{co}(A+D)=\lambda+\operatorname{co}\left(A^{-}\right)-\operatorname{ro}\left(A^{-}\right)$, then $\mathrm{m}^{(A, \lambda)}=\mathrm{m}^{(A+D)}$. Otherwise,

$$
\mathrm{m}^{(A, \lambda)}=\sum_{B \in \Xi_{r}, B \prec A} f_{B, A} \mathrm{~m}^{(B)} \quad\left(f_{B, A} \in \mathbb{Q}(v)\right) .
$$

Proof. If $c o(A+D)=\lambda+\operatorname{co}\left(A^{-}\right)-\operatorname{ro}\left(A^{-}\right)$for some $D \in \Xi^{0}$, then $\lambda=\lambda(A+D)$, and the first assertion follows from the definition. Now suppose that no such $D \in \Xi^{0}$ exists. By 4.14 and $4.9 \mathrm{~m}^{(A, \lambda)}=\mathrm{e}^{\left(A^{+}\right)} \mathrm{f}^{\left(A^{-}\right)} \mathrm{k}_{\lambda^{\prime \prime}}$, with $\lambda^{\prime \prime}=\lambda+\operatorname{co}\left(A^{-}\right)-\operatorname{ro}\left(A^{-}\right)$. By [1, 5.4(c)],

$$
\mathrm{e}^{\left(A^{+}\right)} \mathbf{f}^{\left(A^{-}\right)}=A(\mathbf{0}, r)+\sum_{\substack{\mathbf{j} \in \mathbb{N}^{n}, B \in \Xi^{ \pm} \\ B \prec A}} f_{B, \mathbf{j}, A} B(\mathbf{j}, r)
$$

for $f_{B, \mathbf{j}, A} \in \mathbb{Q}(v)$, where $\Xi_{\leqslant r}^{ \pm}=\left\{A \in \Xi^{ \pm} \mid \sigma(A) \leqslant r\right\}$. By (5.4.1), $\left[\operatorname{diag}\left(\lambda^{\prime \prime}\right)\right]=\mathrm{k}_{\lambda^{\prime \prime}}$, and so $A(\mathbf{0}, r) \mathrm{k}_{\lambda^{\prime \prime}}=0$, since there is no $D \in \Xi^{0}$ satisfying $\operatorname{co}(A+D)=\lambda+\operatorname{co}\left(A^{-}\right)-$ $\operatorname{ro}\left(A^{-}\right)$. Therefore, $\mathrm{m}^{(A, \lambda)}=\sum f_{B, \mathbf{f}, A} B(\mathbf{j}, r) \mathrm{k}_{\lambda^{\prime \prime}}$. But by (5.4.1) and (3.0.1) (see also (3.0.3) $), B(\mathbf{j}, r) \mathrm{k}_{\lambda^{\prime \prime}}$ is equal either to 0 or to $v^{a}[B+D]$ for some $a \in \mathbb{N}$ and some $D \in \Xi^{0}$. The corollary now follows from (5.5.1), using the fact, discussed above, that $A<B \Longrightarrow A \prec B$.

Another monomial basis for $\mathbf{S}_{r}$ results by replacing the divided powers $\mathrm{e}_{i}^{(a)}$ by the ordinary powers $\mathrm{e}_{i}^{a}$. Also, if the $\mathrm{k}_{\lambda}$ in $\mathfrak{M}$ are replaced by the $\mathrm{k}_{\lambda^{\prime}}$ defined in $4.7(2)$, then we obtain a new monomial basis. Monomial bases can be obtained by applying the anti-automorphism $\tau$ defined in 4.2 to the known monomial bases. However, we next show that $\mathfrak{M}$ is an integral basis.

\section{INTEGRAL FORMS AND PBW BASES}

Recall from $\oint 2$ the various $\mathcal{Z}$-integral forms $U^{+}, U^{-}$and $U^{0}$. These subalgebras are all free over $\mathcal{Z}$; see 2.2 for a basis of $U^{0}$. For $U^{+}$and $U^{-}$, the so-called PBW bases are described as follows. Given a reduced expression $w_{0}=s_{i_{1}} s_{i_{2}} \cdots s_{i_{\nu}}$ for 
the longest word $w_{0}$ of $\mathfrak{S}_{n}$, let $\mathbf{i}=\left(i_{1}, i_{2}, \cdots, i_{\nu}\right)$. For any $\mathbf{c}=\left(c_{1}, \cdots, c_{\nu}\right) \in \mathbb{N}^{\nu}$, define

$$
E_{\mathbf{i}}^{\mathbf{c}}=E_{i_{1}}^{\left(c_{1}\right)} \widetilde{T}_{i_{1}}\left(E_{i_{2}}^{\left(c_{2}\right)}\right) \widetilde{T}_{i_{1}} \widetilde{T}_{i_{2}}\left(E_{i_{3}}^{\left(c_{3}\right)}\right) \cdots \widetilde{T}_{i_{1}} \widetilde{T}_{i_{2}} \cdots \widetilde{T}_{i_{\nu-1}}\left(E_{i_{\nu}}^{\left(c_{\nu}\right)}\right),
$$

where the $\widetilde{T}_{i}$ are the braid group actions on $\mathbf{U}[18,1.3]$. Then $\left\{E_{\mathbf{i}}^{\mathbf{c}}\right\}_{\mathbf{c} \in \mathbb{N}^{\nu}}$ is a $\mathcal{Z}$-basis for $U^{+}$. Let $F_{\mathbf{i}}^{\mathbf{c}}=\Omega\left(E_{\mathbf{i}}^{\mathbf{c}}\right)\left(\right.$ cf. $[18,1.3(\mathrm{~d})]$ and $(2.1 .1)$ ); so $\left\{F_{\mathbf{i}}^{\mathbf{c}}\right\}_{\mathbf{c} \in \mathbb{N}^{\nu}}$ is a $\mathcal{Z}$-basis for $U^{-}$.

In [19, 7.8], Lusztig established a relation between the monomial basis given in 3.3 and a PBW basis. To describe this, we choose the following reduced expression (see (3.2.1)):

$$
w_{0}=s_{i_{1}} s_{i_{2}} \cdots s_{i_{\nu}}=\left(s_{n-1} s_{n-2} \cdots s_{1}\right)\left(s_{n-1} s_{n-2} \cdots s_{2}\right) \cdots\left(s_{n-1} s_{n-2}\right) s_{n-1} .
$$

So $\mathbf{i}=(n-1, \cdots, 2,1, \cdots, n-1, n-2, n-1)$. Let $\alpha_{1}, \cdots, \alpha_{n-1}$ be the standard list of simple roots of type $A_{n-1}$, and put $\beta_{1}=\alpha_{n-1}$ and $\beta_{k}=s_{i_{1}} s_{i_{2}} \cdots s_{i_{k-1}}\left(\alpha_{i_{k}}\right)$. Then, ordering from left to right down successive rows, we have the following listing of the positive roots:

$$
\begin{aligned}
\left\{\beta_{1}, \cdots, \beta_{\nu}\right\}=\{ & \alpha_{n-1}, \alpha_{n-1}+\alpha_{n-2}, \cdots, \alpha_{n-1}+\cdots+\alpha_{1}, \\
& \alpha_{n-2}, \alpha_{n-2}+\alpha_{n-3}, \cdots, \alpha_{n-2}+\cdots+\alpha_{1}, \\
& \cdots \\
& \alpha_{2}, \alpha_{2}+\alpha_{1} \\
& \left.\alpha_{1}\right\} .
\end{aligned}
$$

Write $\beta_{k}=\sum_{j=1}^{n-1} p_{j k} \alpha_{j}$, and define $\chi_{\mathbf{i}}: \mathbb{N}^{\nu} \rightarrow \mathbb{N}^{n-1}$ by

$$
\mathbf{c}=\left(c_{1}, c_{2}, \cdots, c_{\nu}\right) \mapsto \chi_{\mathbf{i}}(\mathbf{c})=\mathbf{d}=\left(d_{1}, \cdots, d_{n-1}\right), \quad \text { where } d_{j}=\sum_{k=1}^{\nu} p_{j k} c_{k} .
$$

Clearly, $\chi_{\mathbf{i}}\left(\mathbf{c}^{\prime}+\mathbf{c}^{\prime \prime}\right)=\chi_{\mathbf{i}}\left(\mathbf{c}^{\prime}\right)+\chi_{\mathbf{i}}\left(\mathbf{c}^{\prime \prime}\right)$. Order the fibre $\chi_{\mathbf{i}}^{-1}(\mathbf{d})$ of $\chi_{\mathbf{i}}$ over $\mathbf{d}$ by setting

$$
\mathbf{c}^{\prime}<_{\operatorname{dim}} \mathbf{c}^{\prime \prime} \Longleftrightarrow \operatorname{dim} \mathcal{O}_{\mathbf{c}^{\prime}}<\operatorname{dim} \mathcal{O}_{\mathbf{c}^{\prime \prime}}
$$

where $\mathcal{O}_{\mathbf{c}}$ denotes the orbit of the quiver representation defined by $\mathbf{c}$ (see [19, p. 463]).

Given c $\in \mathbb{N}^{\nu}$, define, for $1 \leqslant k \leqslant \nu, \mathbf{c}_{k} \in \mathbb{N}^{\nu}$ such that the entry in the $k$-th position is $c_{k}$, and 0 otherwise. (Thus, $\mathbf{c}=\sum_{k=1}^{\nu} \mathbf{c}_{k}$.) Put $\mathbf{d}^{k}=\chi_{\mathbf{i}}\left(\mathbf{c}_{k}\right)$, and define

$$
E((\mathbf{c}))=E\left(\mathbf{d}^{1}\right) E\left(\mathbf{d}^{2}\right) \cdots E\left(\mathbf{d}^{\nu}\right), \quad \text { where } E\left(\mathbf{d}^{k}\right)=E_{1}^{\left(d_{1}^{k}\right)} E_{2}^{\left(d_{2}^{k}\right)} \cdots E_{n-1}^{\left(d_{n-1}^{k}\right)} .
$$

Define a bijection $\kappa: \mathbb{N}^{\nu} \rightarrow \Xi^{+}$by sending $\mathbf{c}$ to $A_{\mathbf{c}}^{+}=\left(a_{i, j}\right)$ so that the first $n-1$ components of $\mathbf{c}$ become the $n$-th column reading upwards, and the next $n-2$ components become the $(n-1)$-th column, and so on, i.e.,

$$
c_{1}=a_{n-1, n}, \cdots, c_{n-1}=a_{1, n-1}, c_{n}=a_{n-2, n-1}, \cdots,
$$

and define $\kappa^{-}: \mathbb{N}^{\nu} \rightarrow \Xi^{-}$similarly. Then we have $E^{\left(A_{\mathbf{c}}^{+}\right)}=E((\mathbf{c}))$. Define $F^{\left(A_{\mathbf{c}}^{-}\right)}=F((\mathbf{c})):=\Omega(E((\mathbf{c})))$.

The following result? appears in [19, 7.8(b)].

\footnotetext{
${ }^{9}$ This version is the result of applying the graph automorphism $E_{i} \mapsto E_{n-i+1}$ to Lusztig's version.
} 
Lemma 6.1. Let $\mathbf{i}=(n-1, \cdots, 2,1, \cdots, n-1, n-2, n-1)$ and let $\mathbf{c} \in \mathbb{N}^{\nu}$. For any $\mathbf{c}^{\prime} \in \mathbb{N}^{\nu}$, there exists $h_{\mathbf{c}, \mathbf{c}^{\prime}} \in \mathcal{Z}$ such that

$$
E((\mathbf{c}))=E_{\mathbf{i}}^{\mathbf{c}}+\sum_{\mathbf{c}^{\prime} \in \chi_{\mathbf{i}}^{-1}(\mathbf{d}): \mathbf{c}^{\prime}<\operatorname{dim} \mathbf{c}} h_{\mathbf{c}, \mathbf{c}^{\prime}} E_{\mathbf{i}}^{\mathbf{c}^{\prime}},
$$

where $\mathbf{d}=\chi_{\mathbf{i}}(\mathbf{c})$. A similar result holds for $F((\mathbf{c}))$.

Remarks 6.2. (1) Using the language of quiver representations, $\mathbf{d}=\chi_{\mathbf{i}}(\mathbf{c})$ is the dimension vector of the quiver representation $V_{\mathbf{c}}$ corresponding to $\mathbf{c}$ (see [19. 4.15]). Thus, $\mathbf{c}^{\prime} \in \chi_{\mathbf{i}}^{-1}(\mathbf{d})$ simply means that $V_{\mathbf{c}^{\prime}}$ and $V_{\mathbf{c}}$ have the same dimension vector.

(2) By [4, 7.3(10)] and the remark 4, 7.4], (6.1.1) may be written

$$
E((\mathbf{c}))=E_{\mathbf{i}}^{\mathbf{c}}+\sum_{\mathbf{c}^{\prime} \in \chi_{\mathbf{i}}^{-1}(\mathbf{d}): \mathcal{O}_{\mathbf{c}^{\prime}} \subsetneq \overline{\mathcal{O}}_{\mathbf{c}}} h_{\mathbf{c}, \mathbf{c}^{\prime}} E_{\mathbf{i}}^{\mathbf{c}^{\prime}}=E_{\mathbf{i}}^{\mathbf{c}}+\sum_{\mathbf{c}^{\prime} \in \chi_{\mathbf{i}}^{-1}(\mathbf{d}): A_{\mathbf{c}^{\prime}}^{+} \prec A_{\mathbf{c}}^{+}} h_{\mathbf{c}, \mathbf{c}^{\prime}} E_{\mathbf{i}}^{\mathbf{c}^{\prime}},
$$

where $\overline{\mathcal{O}}_{\mathbf{c}}$ is the Zariski closure of $\mathcal{O}_{\mathbf{c}}$. Here we have used the fact, given in [2, 3.2] (see $[12,5.4]$ for details), that $\mathcal{O}_{\mathbf{c}^{\prime}} \underset{\neq}{\subset \mathcal{O}_{\mathbf{c}}}$ implies $A_{\mathbf{c}^{\prime}}^{+} \prec A_{\mathbf{c}}^{+}$.

Corollary 6.3. The set $\left\{E^{(A)}\right\}_{A \in \Xi^{+}}$(resp., $\left\{F^{(B)}\right\}_{B \in \Xi^{-}}$) forms a basis for $U^{+}$ (resp., $U^{-}$).

By $[9], U_{r}=\zeta_{r}(U)$ is an integral $\mathcal{Z}$-form of $\mathbf{U}_{r}$, generated by $[D],\left(m E_{h, h+1}\right)(\mathbf{0}, r)$ and $\left(m E_{h+1, h}\right)(\mathbf{0}, r)$ with $D \in \Xi_{r}^{0}, m \in \mathbb{N}$.

Theorem 6.4. The $\mathcal{Z}$-subalgebra $U_{r}$ is isomorphic to the subalgebra of $\mathbf{S}_{r}$ generated over $\mathcal{Z}$ by $\mathrm{e}_{i}^{(m)}, \mathbf{f}_{i}^{(m)}(m \in \mathbb{Z}, 1 \leqslant i \leqslant n-1)$ and $\mathrm{k}_{\lambda}(\lambda \in \Lambda(n, r))$. Moreover, the set $\mathfrak{M}$ defined in 4.14 forms a $\mathcal{Z}$-basis for $U_{r}$. (It is called the monomial basis for $U_{r}$.)

Proof. Since $\{[A]\}_{A \in \Xi_{r}}$ forms a $\mathcal{Z}$-basis for $U_{r}$, it follows from 5.5 that $\mathfrak{M}$ forms a $\mathcal{Z}$-basis of $U_{r}$. The first assertion follows as well.

Recall from 4.14 that, for any $A \in \Xi_{r}, \lambda(A)=\left(\sigma_{1}(A), \cdots, \sigma_{n}(A)\right)$. By 4.72 2$)$ and its proof, we have another integral basis for $U_{r}$.

Corollary 6.5. The set

$$
\mathfrak{M}^{\prime}=\left\{\mathrm{e}^{\left(A^{+}\right)} \mathrm{k}_{\lambda(A)}^{\prime} \mathrm{f}^{\left(A^{-}\right)} \mid A \in \Xi_{r}\right\}
$$

forms a $\mathcal{Z}$-basis for $U_{r}$.

Let $\mathrm{e}_{\mathbf{i}}^{\mathbf{c}}=\zeta_{r}\left(E_{\mathbf{i}}^{\mathbf{c}}\right)$ and $\mathbf{f}_{\mathbf{i}}^{\mathbf{c}}=\zeta_{r}\left(F_{\mathbf{i}}^{\mathbf{c}}\right)$. For any $A \in \Xi_{r}$, let $\mathbf{c}\left(A^{+}\right) \in \mathbb{N}^{\nu}$ (resp., $\left.\mathbf{c}\left(A^{-}\right) \in \mathbb{N}^{\nu}\right)$ correspond to $A^{+}$(resp., $A^{-}$) under the bijection $\kappa$ (resp., $\kappa^{-}$) above. We now obtain the PBW-basis for $U_{r}$.

Theorem 6.6. Let $\mathbf{i}=(n-1, \cdots, 2,1, \cdots, n-1, n-2, n-1)$. Then the set

$$
\mathfrak{B}_{\mathbf{i}}=\left\{\mathrm{e}_{\mathbf{i}}^{\mathbf{c}\left(A^{+}\right)} \mathrm{k}_{\lambda(A)} \mathbf{f}_{\mathbf{i}}^{\mathbf{c}\left(A^{-}\right)} \mid A \in \Xi_{r}\right\}
$$

forms a $\mathcal{Z}$-basis for $U_{r}$.

Proof. Using 6.1 and noting 4.11 and 6.3. we may write $\mathrm{e}_{\mathbf{i}}^{\mathbf{c}\left(A^{+}\right)}=\mathrm{e}^{\left(A^{+}\right)}+$lower terms and $\mathrm{f}_{\mathrm{i}}^{\mathbf{c}\left(A^{-}\right)}=\mathrm{f}^{\left(A^{-}\right)}+$lower terms. Here the lower terms are relative to $\preccurlyeq$ by 6.2 (2). By 6.4, the coefficients $f_{B, A}$ in 5.6 must lie in $\mathcal{Z}$. So (5.6.1) gives

$$
e_{\mathbf{i}}^{\mathbf{c}\left(A^{+}\right)} \mathbf{k}_{\lambda(A)} \mathbf{f}_{\mathbf{i}}^{\mathbf{c}\left(A^{-}\right)}=\mathrm{m}^{(A)}+\text { lower terms (relative to } \preccurlyeq \text { ). }
$$


Now the assertion follows from 6.4.

Corollary 6.7. Maintain the notation used above. The set

$$
\mathfrak{B}_{\mathbf{i}}^{\prime}=\left\{\mathrm{e}_{\mathbf{i}}^{\mathbf{c}\left(A^{+}\right)} \mathbf{k}_{\lambda(A)}^{\prime} \mathbf{f}_{\mathbf{i}}^{\mathbf{c}\left(A^{-}\right)} \mid A \in \Xi_{r}\right\}
$$

forms a $\mathcal{Z}$-basis for $U_{r}$.

\section{The TRANSFER MAPS $U_{n+r} \rightarrow U_{r}$}

We define epimorphisms $U_{n+r} \rightarrow U_{r}$; these are the "transfer maps" in [21. Let $\mathbf{k}_{i}, \mathbf{e}_{i}$ and $\mathbf{f}_{i}$ denote the generators for $\mathbf{U}_{n+r}$. Denote the monomial basis for $\mathbf{U}_{n+r}$ by $\left\{\mathbf{m}^{(A)}\right\}_{A \in \Xi_{n+r}}$.

Proposition 7.1. There is a unique algebra epimorphism

$$
\psi=\psi_{n+r, r}: \mathbf{U}_{n+r} \rightarrow \mathbf{U}_{r}
$$

satisfying $\psi\left(\mathbf{k}_{i}\right)=v \mathbf{k}_{i}, \psi\left(\mathbf{e}_{i}\right)=\mathbf{e}_{i}$ and $\psi\left(\mathbf{f}_{i}\right)=\mathbf{f}_{i}$.

Proof. It follows directly that $\psi$ preserves the relations for $\mathbf{k}_{i}, \mathbf{e}_{i}$ and $\mathbf{f}_{i}$.

The maps $\psi_{n+r, r}$ agree with the maps $\phi_{n+r, r}$, described (for both finite and affine cases) in [20, 9.1]. The existence of $\phi_{n+r, r}$ is proved in [21, 1.10] (cf. [10, 5.4(a)] for a dual treatment in the $G L_{n}$ case). Our next result shows that $\mathfrak{M}$ shares a similar property with the canonical basis under the transfer maps. See the conjecture [20] $9.2]$ and a proof in [22].

Corollary 7.2. The map $\psi$ induces an epimorphism $\psi: U_{n+r} \rightarrow U_{r}$. More precisely,

$$
\psi\left(\mathbf{m}^{(A)}\right)= \begin{cases}\mathrm{m}^{\left(A-I_{n}\right)}, & \text { if } A-I_{n} \in \Xi_{r}, \\ 0, & \text { otherwise. }\end{cases}
$$

Here $I_{n}$ denotes the $n \times n$ identity matrix.

Proof. We first observe that, if $\lambda_{i} \geqslant 1$, then

$$
\left[\begin{array}{c}
v \mathrm{k}_{i} ; 0 \\
\lambda_{i}
\end{array}\right]=\frac{v \mathrm{k}_{i}-v^{-1} \mathrm{k}_{i}^{-1}}{v^{\lambda_{i}}-v^{-\lambda_{i}}}\left[\begin{array}{c}
\mathrm{k}_{i} ; 0 \\
\lambda_{i}-1
\end{array}\right] .
$$

Let $\mathbf{1}=(1, \cdots, 1) \in \mathbb{N}^{n}$. For any $\lambda \in \Lambda(n, n+r)$, if $\lambda-\mathbf{1} \in \Lambda(n, r)$, then $\lambda_{i} \geqslant 1$ for all $i$ and, by $4.5(2)$,

$$
\psi\left(\mathbf{k}_{\lambda}\right)=\prod_{i=1}^{n} \frac{v \mathbf{k}_{i}-v^{-1} \mathbf{k}_{i}^{-1}}{v^{\lambda_{i}}-v^{-\lambda_{i}}} \mathbf{k}_{\lambda-\mathbf{1}}=\mathbf{k}_{\lambda-\mathbf{1}} .
$$

If $\lambda-\mathbf{1} \notin \Lambda(n, r)$, then we have clearly $\psi\left(\mathbf{k}_{\lambda}\right)=\mathbf{k}_{\mu}$ with $|\mu|=n+r-x>r$, where $x$ is the number of $i$ with $\lambda_{i}=0$. Therefore, $\psi\left(\mathbf{k}_{\lambda}\right)=0$. The rest of the proof is clear. 


\section{Presentations for Borel subalgebras}

For positive integers $n$ and $r$, the symmetric group $\mathfrak{S}_{r}$ acts on the set

$$
\mathcal{I}=\mathcal{I}(n, r):=\left\{\left(i_{1}, \cdots, i_{r}\right) \mid 1 \leqslant i_{j} \leqslant n\right\}
$$

by place permutations, and then acts on $\mathcal{I} \times \mathcal{I}$ diagonally.

There is a bijection between $\Xi_{r}$ and the set of all $\mathfrak{S}_{r}$-orbits in $\mathcal{I} \times \mathcal{I}$ defined as follows: If $A=\left(a_{i, j}\right) \in \Xi_{r}$ with $\lambda=\operatorname{ro}(A)$, we let

$$
\mathbf{i}_{A}=(\underbrace{1, \cdots, 1}_{\lambda_{1} \text { times }}, \cdots, \underbrace{n, \cdots, n}_{\lambda_{n} \text { times }}) \text { and } \mathbf{j}_{A}=\left(\mathbf{j}_{1}, \cdots, \mathbf{j}_{n}\right),
$$

where $\mathbf{j}_{i}=(\underbrace{1, \cdots, 1}_{a_{i, 1} \text { times }}, \cdots, \underbrace{n, \cdots, n}_{a_{i, n} \text { times }})$. The map sending $A$ to the orbit containing $\left(\mathbf{i}_{A}, \mathbf{j}_{A}\right)$ is a bijection.

We order $\mathcal{I}$ by setting $\mathbf{i} \leqslant \mathbf{j}$ if and only if $i_{1} \leqslant j_{1}, \ldots, i_{r} \leqslant j_{r}$. Clearly, $A \in \Xi_{r}$ with $A^{-}=0 \Longleftrightarrow \mathbf{i}_{A} \leqslant \mathbf{j}_{A}$. It is known (see, e.g., [13, 1.3.3,5.6.1]) that the subspace $\mathbf{U}_{r}^{\geqslant 0}$ (resp., $\mathbf{U}_{r}^{\leqslant 0}$ ) spanned by all $[A]$ where $A \in \Xi_{r}$ with $A^{-}=0$ (resp., $\left.A^{+}=0\right)$ is a subalgebra, called a Borel subalgebra. Clearly, we have the following dimension formula:

$$
\operatorname{dim} \mathbf{U}_{r}^{\geqslant 0}=\operatorname{dim} \mathbf{U}_{r}^{\leqslant 0}=\left(\begin{array}{c}
r+\left(\begin{array}{c}
n+1 \\
2 \\
r
\end{array}\right)-1
\end{array}\right)=\sum_{\lambda \in \Lambda(n, r)} \prod_{i=1}^{n}\left(\begin{array}{c}
\lambda_{i}+i-1 \\
i-1
\end{array}\right) .
$$

We now can state the following.

Theorem 8.1. The subalgebra $\mathbf{U}_{r}^{\geqslant 0}$ is isomorphic to the algebra $\mathbf{B}$ with generators $\mathrm{e}_{i}, \mathrm{k}_{i},(1 \leqslant i, j \leqslant n-1)$, subject to the following relations:

(a) $\mathrm{k}_{i} \mathrm{k}_{j}=\mathrm{k}_{j} \mathrm{k}_{i}$

(b) $\left[\mathrm{k}_{1} ; t_{1}\right]^{!}\left[\mathrm{k}_{2} ; t_{2}\right]^{!} \cdots\left[\mathrm{k}_{n-1} ; t_{n-1}\right]^{!}=0, \forall t_{i} \in \mathbb{N}, t_{1}+\cdots+t_{n-1}=r+1$;

(c) $\mathrm{e}_{i} \mathrm{e}_{j}=\mathrm{e}_{j} \mathrm{e}_{i} \quad(|i-j|>1)$;

(d) $\mathrm{e}_{i}^{2} \mathrm{e}_{j}-\left(v+v^{-1}\right) \mathbf{e}_{i} \mathrm{e}_{j} \mathrm{e}_{i}+\mathrm{e}_{j} \mathrm{e}_{i}^{2}=0$ when $|i-j|=1$;

(e) $\mathrm{k}_{i} \mathrm{e}_{j}=v^{\epsilon(i, j)} \mathrm{e}_{j} \mathrm{k}_{i}$, where $\epsilon(i, i)=1, \epsilon(i+1, i)=-1$ and $\epsilon(i, j)=0$, otherwise.

A similar result holds for $\mathbf{U}_{r}^{\leqslant 0}$.

Proof. Identifying $\mathbf{U}_{r}$ with $\mathbf{S}_{r}$, it is clear that $\mathbf{U}_{r}^{\geqslant 0}$ is generated by the $\mathrm{e}_{i}, 1 \leqslant i<n$, and the $\mathrm{k}_{\lambda}, \lambda \in \Lambda(n, r)$. Using [5.5, $\mathbf{U}_{r}^{\geqslant 0}$ has a basis consisting of terms $\mathrm{e}^{\left(A^{+}\right)} \mathrm{k}_{\lambda}$, with $\lambda \in \Lambda(n, r)$, and $\lambda_{i} \geq \sigma_{i}\left(A^{+}\right), \forall i$. Temporarily denote the generators of $\mathbf{B}$ by $\mathrm{e}_{i}^{\prime}, \mathrm{k}_{i}^{\prime}$. Because 4.10 (2) clearly holds for the algebra $\mathbf{B}$, it is obviously spanned by elements $\mathrm{e}^{\prime\left(A^{+}\right)} \mathrm{k}_{\lambda}^{\prime}$ with $A^{+}$, etc., satisfying the same conditions for the basis vectors $\mathrm{e}^{\left(A^{+}\right)} \mathrm{k}_{\lambda}$ of $\mathbf{U}_{r}^{\geqslant 0}$. Hence, the natural algebra surjection $\mathbf{B} \rightarrow \mathbf{U}_{r}^{\geqslant 0}$ is an isomorphism by dimension considerations.

Let $U_{r}^{\geqslant 0}$ (resp., $U_{r}^{\leqslant 0}$ ) be the $\mathcal{Z}$-subalgebra of $\mathbf{U}_{r}$ generated by the $\mathrm{e}_{i}^{(m)}$ (resp., $\left.\mathrm{f}_{i}^{(m)}\right), \mathrm{k}_{\lambda}$ with $m \in \mathbb{N}, \lambda \in \Lambda(n, r)$.

Corollary 8.2. The set of all $\mathrm{e}^{(A)} \mathrm{k}_{\lambda}$ (resp., $\left.\mathrm{k}_{\lambda} \mathrm{f}^{(A)}\right)$, where $A \in \Xi^{+}$(resp., $A \in$ $\left.\Xi^{-}\right), \lambda \in \Lambda(n, r)$ satisfying $\lambda_{i} \geqslant \sigma_{i}(A), \forall i$, forms a $\mathcal{Z}$-basis for $U_{r}^{\geqslant 0}$ (resp., $U_{r}^{\leqslant 0}$ ).

We have a further decomposition for $U_{r}, U_{r}^{\geqslant 0}$ and $U_{r}^{\leqslant 0}$. Let $U_{r}^{+}$(resp., $U_{r}^{-}$, $U_{r}^{0}$ ) be the $\mathcal{Z}$-subalgebras of $U_{r}$ generated by the $\mathrm{e}_{i}^{(m)}$ (resp., $\mathbf{f}_{i}^{(m)}, \mathrm{k}_{\lambda}$ ). Note that 
$U_{r}^{0} \otimes \mathcal{Z} \mathbb{Q}(v)=\mathbf{U}_{r}^{0}=\mathbf{S}_{r}^{0}$. A PBW basis version of the following is obtained in [14] $2.5-6]$.

Theorem 8.3. The algebra $U_{r}^{+}$(resp., $U_{r}^{-}$) is $\mathcal{Z}$-free with basis

$$
\left\{\mathrm{e}^{(A)} \mid A \in \Xi^{+}, \sigma(A) \leqslant r\right\} \quad\left(\text { resp. },\left\{\mathrm{f}^{(A)} \mid A \in \Xi^{-}, \sigma(A) \leqslant r\right\}\right) .
$$

Hence, $\operatorname{dim} \mathbf{S}_{r}^{+}=\operatorname{dim} \mathbf{S}_{r}^{-}=\left(\begin{array}{c}N+r \\ r\end{array}\right)$, where $N=\frac{n(n-1)}{2}$. Moreover, we have triangular decompositions:

$$
U_{r}=U_{r}^{+} U_{r}^{0} U_{r}^{-}, \quad U_{r}^{\geqslant 0}=U_{r}^{+} U_{r}^{0}, \text { and } U_{r}^{\leqslant 0}=U_{r}^{0} U_{r}^{-} .
$$

Proof. By 4.11 the two sets span $U_{r}^{+}$and $U_{r}^{-}$, respectively. The linear independence is seen easily from 8.2 by writing $\mathrm{e}^{(A)}=\sum_{\lambda: \lambda_{i} \geqslant \sigma_{i}(A)} \mathrm{e}^{(A)} \mathrm{k}_{\lambda}$.

Note that, by 4.12, the multiplication map from $U_{r}^{+} \otimes U_{r}^{0} \otimes U_{r}^{-}$to $U_{r}$ is no longer injective. Thus, there is no tensor product triangular decomposition in this case.

\section{IDENTIFYING THE MONOMIAL BASIS IN $H$}

In this section, assume that $n=r$ for simplicity; all results below are still valid for $r \leqslant n$. Let

$$
\omega=(1,1, \cdots, 1) \in \Lambda(n, n),
$$

and let $\mathbf{H}=\mathrm{k}_{\omega} \mathbf{U}_{n} \mathrm{k}_{\omega}$ and $H=\mathrm{k}_{\omega} U_{n} \mathrm{k}_{\omega}$. By (5.4.1), $\mathrm{k}_{\omega}=\left[I_{n}\right]$, where $I_{n}$ is the $n \times n$ identity matrix. It is well known (see, e.g., [6]) that $H$ is isomorphic to the Hecke algebra defined in (1.0.1). However, in this section, we will not assume this identification, but rederive it from the monomial basis theory for $q$-Schur algebras. At a deeper and more interesting level, 9.4 explicitly identifies a basis $\mathfrak{M}_{\omega} \subset \mathfrak{M}$ of $H \subset U_{n}$ as a monomial basis involving certain monomials in the Kazhdan-Lusztig elements $C_{s}^{\prime}$; see 9.6 (2).

For $w \in \mathfrak{S}_{n}$, the permutation matrix $A_{w} \in \Xi_{n}$ is defined inductively by setting $A_{w}=A_{y} A_{s}$, where $w=y s$ with $y<w$ and $s=(i, i+1)$ for some $i$. Writing $\mathrm{m}^{\left(A_{w}\right)}=\mathrm{e}^{\left(A_{w}^{+}\right)} \mathrm{k}_{\lambda} \mathrm{f}^{\left(A_{w}^{-}\right)}$as in 4.144.9 implies that $\mathrm{e}^{\left(A_{w}^{+}\right)} \mathrm{k}_{\lambda}=\mathrm{k}_{\omega} \mathrm{e}^{\left(A_{w}^{+}\right)}$and $\mathrm{k}_{\lambda} \mathrm{f}^{\left(A_{w}^{-}\right)}=$ $\mathbf{f}^{\left(A_{w}^{-}\right)} \mathrm{k}_{\omega} ;$ so $\mathrm{m}^{\left(A_{w}\right)} \in \mathbf{H}$.

Proposition 9.1. The algebra $H$ is free over $\mathcal{Z}$ with basis $\mathfrak{M}_{\omega}=\left\{\mathrm{m}^{\left(A_{w}\right)} \mid w \in \mathfrak{S}_{n}\right\}$.

Proof. For $A \in \Xi_{n}$, if $\mathrm{k}_{\omega}[A] \mathrm{k}_{\omega} \neq 0$, then $\operatorname{ro}(A)=c o(A)=\omega$. So $A$ is necessarily a permutation matrix. Thus, $\mathfrak{M}_{\omega} \subseteq \mathrm{k}_{\omega} \mathfrak{M k}_{\omega}$. By [5.5] if $A$ is not a permutation matrix and $\mathrm{k}_{\omega} \mathrm{m}^{(A)} \mathrm{k}_{\omega} \neq 0$, then $\mathrm{k}_{\omega} \mathrm{m}^{(A)} \mathrm{k}_{\omega}$ is a linear combination of the elements of $\mathfrak{M}_{\omega}$. Therefore, $\mathfrak{M}_{\omega}$ forms a basis for $H$.

We record the following simple commutation relations.

Lemma 9.2. Let $\mathrm{m}$ be a monomial in the $\mathrm{f}_{i}$. For any $1 \leqslant i \leqslant n-2$, let $\partial_{i}(\mathrm{~m})=$ $2 \operatorname{deg}_{i}(\mathrm{~m})-\operatorname{deg}_{i-1}(\mathrm{~m})-\operatorname{deg}_{i+1}(\mathrm{~m})$, where $\operatorname{deg}_{j}$ denotes the degree of $\mathrm{f}_{j}$ in $\mathrm{m}$. Then we have the following.

(1) If $\partial_{i}(\mathrm{~m})=1$, then $\left[\begin{array}{c}\tilde{\mathrm{k}}_{i} ; 0 \\ 1\end{array}\right] \mathrm{mk}_{\omega}=-\mathrm{mk}_{\omega}$.

(2) If $\mathrm{m}=\mathrm{m}_{1} \mathrm{f}_{i} \mathrm{~m}_{2}$ and $\partial_{i}\left(\mathrm{~m}_{2}\right)=0$, then $\mathrm{m}_{1} \mathrm{f}_{i} \mathrm{e}_{i} \mathrm{~m}_{2} \mathrm{k}_{\omega}=\mathrm{m}_{1} \mathrm{e}_{i} \mathrm{f}_{i} \mathrm{~m}_{2} \mathrm{k}_{\omega}$.

Proof. Since $\left[\begin{array}{c}\tilde{\mathrm{k}}_{i} ; 0 \\ 1\end{array}\right] \mathbf{f}_{i}=\mathbf{f}_{i}\left[\begin{array}{c}\tilde{\mathrm{k}}_{i} ;-2 \\ 1\end{array}\right]$ and $\left[\begin{array}{c}\tilde{\mathrm{k}}_{i} ; 0 \\ 1\end{array}\right] \mathbf{f}_{j}=\mathbf{f}_{j}\left[\begin{array}{c}\tilde{\mathrm{k}}_{i} ; 1 \\ 1\end{array}\right]$ for $j=i-1, i+1$, it follows that $\left[\begin{array}{c}\tilde{\mathrm{k}}_{i} ; 0 \\ 1\end{array}\right] \mathrm{m}=\mathrm{m}\left[\begin{array}{c}\tilde{\mathrm{k}}_{i} ;-\partial_{i}(\mathrm{~m}) \\ 1\end{array}\right]$. Since $\left[\begin{array}{c}\tilde{\mathrm{k}}_{i} ; c \\ 1\end{array}\right] \mathbf{k}_{\omega}=c \mathbf{k}_{\omega}$ for $c=0,-1$ and $\mathbf{f}_{i} \mathbf{e}_{i}=\mathrm{e}_{i} \mathbf{f}_{i}-\left[\begin{array}{c}\tilde{\mathbf{k}}_{i} ; 0 \\ 1\end{array}\right]$, the two assertions follow immediately. 
Let $s_{i}=(i, i+1)$ and put

$$
\mathrm{C}_{i}=\mathrm{m}^{\left(A_{s_{i}}\right)}=\mathrm{e}^{\left(A_{s_{i}}^{+}\right)} \mathrm{k}_{\lambda\left(s_{i}\right)} \mathrm{f}^{\left(A_{s_{i}}^{-}\right)}=\mathrm{e}_{i} \mathrm{k}_{\lambda\left(s_{i}\right)} \mathrm{f}_{i}=\mathrm{k}_{\omega} \mathrm{e}_{i} \mathrm{f}_{i} \mathrm{k}_{\omega}=\mathrm{k}_{\omega} \mathrm{f}_{i} \mathrm{e}_{i} \mathrm{k}_{\omega}
$$

where

$$
\lambda\left(s_{i}\right)=\lambda\left(A_{s_{i}}\right)=\left(\sigma_{1}\left(A_{s_{i}}\right), \cdots, \sigma_{n}\left(A_{s_{i}}\right)\right)=(1, \cdots, 1, \underset{(i)}{0}, 2,1 \cdots, 1)
$$

as defined in 4.14 For any $i$ we have $\mathrm{k}_{\omega} \mathrm{e}_{i}=\mathrm{e}_{i} \mathrm{k}_{\lambda\left(s_{i}\right)}$ by 4.8 since $\lambda\left(s_{i}\right)=\omega+\alpha_{i}$.

Theorem 9.3. The elements $\mathrm{T}_{i}:=\mathrm{C}_{i}-v^{-1}, 1 \leqslant i \leqslant n-1$, satisfy the following relations:

(a) $\left(\mathrm{T}_{i}-v\right)\left(\mathrm{T}_{i}+v^{-1}\right)=0$;

(b) $\mathrm{T}_{i} \mathrm{~T}_{j}=\mathrm{T}_{j} \mathrm{~T}_{i}$ when $|i-j|>1$;

(c) $\mathrm{T}_{i} \mathrm{~T}_{i+1} \mathrm{~T}_{i}=\mathrm{T}_{i+1} \mathrm{~T}_{i} \mathrm{~T}_{i+1}$ when $1 \leqslant i \leqslant n-2$.

In particular, $\mathrm{T}_{i}$ is invertible and $\mathrm{T}_{i}^{-1}=\mathrm{C}_{i}-v$.

Proof. If $\mathrm{T}_{i}^{-1}$ has the required form, then (a) follows. So, to prove (a), we must show that

This is clear, since

$$
\left(\mathrm{C}_{i}-v^{-1}\right)\left(\mathrm{C}_{i}-v\right)=1 .
$$

$$
\begin{aligned}
\mathrm{C}_{i}^{2} & =\left(\mathrm{e}_{i} \mathrm{k}_{\lambda\left(s_{i}\right)} \mathbf{f}_{i}\right)\left(\mathbf{e}_{i} \mathrm{k}_{\lambda\left(s_{i}\right)} \mathbf{f}_{i}\right)=\mathbf{e}_{i} \mathrm{k}_{\lambda\left(s_{i}\right)}\left(\mathbf{e}_{i} \mathbf{f}_{i}-\frac{\tilde{\mathbf{k}}_{i}-\tilde{\mathbf{k}}_{i}^{-1}}{v-v^{-1}}\right) \mathbf{k}_{\lambda\left(s_{i}\right)} \mathbf{f}_{i} \\
& =\left(v+v^{-1}\right) \mathbf{e}_{i} \mathrm{k}_{\lambda\left(s_{i}\right)} \mathbf{f}_{i}=\left(v+v^{-1}\right) \mathbf{C}_{i} .
\end{aligned}
$$

Here we use 4.5 (2) and the fact that, since the $i$-th component of $\lambda\left(s_{i}\right)$ is $0,4.12$ (1) implies $\mathrm{k}_{\lambda\left(s_{i}\right)} \mathrm{e}_{i}=0$. The relation (b) follows easily from the relation $\mathrm{C}_{i} \mathrm{C}_{j}=\mathrm{C}_{j} \mathrm{C}_{i}$ whenever $|i-j|>1$. We now prove (c). Since

$$
\mathrm{T}_{i} \mathrm{~T}_{i+1} \mathrm{~T}_{i}=\mathrm{C}_{i} \mathrm{C}_{i+1} \mathrm{C}_{i}-\mathrm{C}_{i}-v^{-1}\left(\mathrm{C}_{i} \mathrm{C}_{i+1}+\mathrm{C}_{i+1} \mathrm{C}_{i}\right)+v^{-2}\left(\mathrm{C}_{i}+\mathrm{C}_{i+1}\right)+v^{-3},
$$

and a similar formula holds with $i$ and $i+1$ switched, it suffices to prove that

$$
\mathrm{C}_{i} \mathrm{C}_{i+1} \mathrm{C}_{i}-\mathrm{C}_{i}=\mathrm{C}_{i+1} \mathrm{C}_{i} \mathrm{C}_{i+1}-\mathrm{C}_{i+1} .
$$

For notational simplicity, put $C=\mathrm{C}_{i} \mathrm{C}_{i+1} \mathrm{C}_{i}$ and $C^{\prime}=\mathrm{C}_{i+1} \mathrm{C}_{i} \mathrm{C}_{i+1}$. Then

$$
C=\mathrm{k}_{\omega}\left(\mathrm{e}_{i} \mathrm{f}_{i} \mathrm{e}_{i+1} \mathrm{f}_{i+1} \mathrm{e}_{i} \mathrm{f}_{i}\right) \mathrm{k}_{\omega}, \quad C^{\prime}=\mathrm{k}_{\omega}\left(\mathrm{e}_{i+1} \mathrm{f}_{i+1} \mathrm{e}_{i} \mathrm{f}_{i} \mathrm{e}_{i+1} \mathrm{f}_{i+1}\right) \mathrm{k}_{\omega} .
$$

Now, by 4.1

$$
\begin{aligned}
\mathrm{e}_{i} \mathrm{f}_{i} \mathrm{e}_{i+1} \mathrm{f}_{i+1} \mathrm{e}_{i} \mathrm{f}_{i} & =\mathrm{e}_{i} \mathrm{e}_{i+1}\left(\mathrm{f}_{i} \mathrm{e}_{i}\right) \mathrm{f}_{i+1} \mathrm{f}_{i}=\mathrm{e}_{i} \mathrm{e}_{i+1}\left(\mathrm{e}_{i} \mathrm{f}_{i}-\left[\begin{array}{c}
\tilde{\mathrm{k}}_{i} ; 0 \\
1
\end{array}\right]\right) \mathrm{f}_{i+1} \mathrm{f}_{i} \\
& =\mathrm{e}_{i} \mathrm{e}_{i+1} \mathrm{e}_{i} \mathrm{f}_{i} \mathrm{f}_{i+1} \mathrm{f}_{i}-\left[\begin{array}{c}
\tilde{\mathrm{k}}_{i} ;-1 \\
1
\end{array}\right] \mathrm{e}_{i} \mathrm{e}_{i+1} \mathrm{f}_{i+1} \mathrm{f}_{i} .
\end{aligned}
$$

By the quantum Serre relations,

$$
\begin{aligned}
\mathrm{e}_{i} \mathrm{e}_{i+1} \mathrm{e}_{i} \mathrm{f}_{i} \mathrm{f}_{i+1} \mathrm{f}_{i}=\left(\mathrm{e}_{i}^{(2)} \mathrm{e}_{i+1}+\mathrm{e}_{i+1} \mathrm{e}_{i}^{(2)}\right)\left(\mathrm{f}_{i}^{(2)} \mathrm{f}_{i+1}+\mathrm{f}_{i+1} \mathrm{f}_{i}^{(2)}\right) \\
\quad=\mathrm{e}_{i}^{(2)} \mathrm{e}_{i+1}\left(\mathrm{f}_{i}^{(2)} \mathrm{f}_{i+1}+\mathrm{f}_{i+1} \mathrm{f}_{i}^{(2)}\right)+\mathrm{e}_{i+1} \mathrm{e}_{i}^{(2)}\left(\mathrm{f}_{i}^{(2)} \mathrm{f}_{i+1}+\mathrm{f}_{i+1} \mathrm{f}_{i}^{(2)}\right) .
\end{aligned}
$$

Since $\mathbf{k}_{\omega} \mathrm{e}_{i}^{(2)}=0=\mathbf{k}_{\omega} \mathrm{e}_{i+1} \mathrm{e}_{i}^{(2)}$ by $4.12(1)$, multiplying by $\mathbf{k}_{\omega}$ gives

$$
C=-\mathrm{k}_{\omega}\left[\begin{array}{c}
\tilde{\mathrm{k}}_{i} ;-1 \\
1
\end{array}\right] \mathrm{e}_{i} \mathrm{e}_{i+1} \mathrm{f}_{i+1} \mathrm{f}_{i} \mathrm{k}_{\omega}=\mathrm{k}_{\omega} \mathrm{e}_{i} \mathrm{e}_{i+1} \mathrm{f}_{i+1} \mathrm{f}_{i} \mathrm{k}_{\omega} .
$$


The calculation of $C^{\prime}$ can be done similarly by switching the subscripts $i$ and $i+1$; so

$$
\begin{aligned}
& \mathrm{e}_{i+1} \mathrm{e}_{i} \mathrm{e}_{i+1} \mathrm{f}_{i+1} \mathrm{f}_{i} \mathrm{f}_{i+1}=\left(\mathrm{e}_{i+1}^{(2)} \mathrm{e}_{i}+\mathrm{e}_{i} \mathrm{e}_{i+1}^{(2)}\right)\left(\mathrm{f}_{i+1}^{(2)} \mathrm{f}_{i}+\mathrm{f}_{i} \mathrm{f}_{i+1}^{(2)}\right) \\
& =\mathrm{e}_{i+1}^{(2)} \mathrm{e}_{i}\left(\mathrm{f}_{i+1}^{(2)} \mathrm{f}_{i}+\mathrm{f}_{i} \mathrm{f}_{i+1}^{(2)}\right)+\mathrm{e}_{i} \mathrm{e}_{i+1}^{(2)} \mathrm{f}_{i} \mathrm{f}_{i+1}^{(2)}+\mathrm{e}_{i} \mathrm{e}_{i+1}^{(2)} \mathrm{f}_{i+1}^{(2)} \mathrm{f}_{i} .
\end{aligned}
$$

However, in this case, only the first two summands vanish after multiplying by $\mathrm{k}_{\omega}$. Thus,

$$
C^{\prime}=\mathrm{k}_{\omega}\left(\mathrm{e}_{i} \mathrm{e}_{i+1}^{(2)} \mathbf{f}_{i+1}^{(2)} \mathbf{f}_{i}-\left[\begin{array}{c}
\tilde{\mathrm{k}}_{i} ;-1 \\
1
\end{array}\right] \mathrm{e}_{i+1} \mathrm{e}_{i} \mathrm{f}_{i} \mathrm{f}_{i+1}\right) \mathrm{k}_{\omega} .
$$

Now, by 2.3 (7) and the last relation in 4.1 , we have

$$
\begin{aligned}
\mathrm{e}_{i}\left(\mathrm{e}_{i+1}^{(2)}\right. & \left.\mathrm{f}_{i+1}^{(2)}\right) \mathbf{f}_{i} \\
& =\mathrm{e}_{i}\left(\mathbf{f}_{i+1}^{(2)} \mathrm{e}_{i+1}^{(2)}+\mathbf{f}_{i+1}\left[\begin{array}{c}
\tilde{\mathrm{k}}_{i+1} ;-2 \\
1
\end{array}\right] \mathrm{e}_{i+1}+\left[\begin{array}{c}
\tilde{\mathrm{k}}_{i+1} ; 0 \\
2
\end{array}\right]\right) \mathbf{f}_{i} \\
& =\mathrm{e}_{i} \mathrm{f}_{i+1}^{(2)} \mathrm{e}_{i+1}^{(2)} \mathrm{f}_{i}+\left[\begin{array}{c}
\tilde{\mathrm{k}}_{i+1} ; 1 \\
2
\end{array}\right] \mathrm{e}_{i} \mathrm{f}_{i}+\left[\begin{array}{c}
\tilde{\mathrm{k}}_{i+1} ; 1 \\
1
\end{array}\right] \mathrm{e}_{i}\left(\mathrm{e}_{i+1} \mathrm{f}_{i+1}-\left[\begin{array}{c}
\tilde{\mathrm{k}}_{i+1} ; 0 \\
1
\end{array}\right]\right) \mathbf{f}_{i} .
\end{aligned}
$$

The first two terms vanish after multiplying by $\mathrm{k}_{\omega}$. The last two terms equal

$$
\left[\begin{array}{c}
\tilde{\mathrm{k}}_{i+1} ; 1 \\
1
\end{array}\right] \mathrm{e}_{i} \mathbf{e}_{i+1} \mathbf{f}_{i+1} \mathbf{f}_{i}-\left[\begin{array}{c}
\tilde{\mathrm{k}}_{i+1} ; 1 \\
1
\end{array}\right]^{2} \mathrm{e}_{i} \mathbf{f}_{i} .
$$

On the other hand, we have

$$
\mathrm{e}_{i+1} \mathrm{e}_{i} \mathbf{f}_{i} \mathrm{f}_{i+1}=\mathrm{e}_{i+1}\left(\mathbf{f}_{i} \mathrm{e}_{i}+\left[\begin{array}{c}
\tilde{\mathrm{k}}_{i} ; 0 \\
1
\end{array}\right]\right) \mathbf{f}_{i+1}=\mathrm{e}_{i+1} \mathbf{f}_{i} \mathrm{e}_{i} \mathbf{f}_{i+1}+\left[\begin{array}{c}
\tilde{\mathrm{k}}_{i} ; 1 \\
1
\end{array}\right] \mathrm{e}_{i+1} \mathbf{f}_{i+1} .
$$

Since $\mathrm{k}_{\omega} \mathrm{e}_{i+1} \mathrm{f}_{i} \mathrm{e}_{i} \mathrm{f}_{i+1} \mathrm{k}_{\omega}=0$, we obtain after combining everything that

$$
C^{\prime}=\mathrm{k}_{\omega} \mathrm{e}_{i} \mathrm{e}_{i+1} \mathrm{f}_{i+1} \mathrm{f}_{i} \mathrm{k}_{\omega}-\mathrm{C}_{i}+\mathrm{C}_{i+1}=C-\mathrm{C}_{i}+\mathrm{C}_{i+1} .
$$

This proves (9.3.1), and hence (c).

Interestingly, the commuting relations $4.1(\mathrm{~g})$ and the quantum Serre relations 4.1(d), (e) give rise to the braid relation 2.3(c). We also record the following relations on the elements $\mathrm{C}_{i}$ (cf. [8, (H1-3)] and [24, §2]):

$$
\left\{\begin{array}{l}
(1) \quad \mathrm{C}_{i}^{2}=\left(v+v^{-1}\right) \mathrm{C}_{i} ; \\
(2) \quad \mathrm{C}_{i} \mathrm{C}_{j}=\mathrm{C}_{j} \mathrm{C}_{i} \text { for }|i-j|>1 \\
(3) \quad \mathrm{C}_{i} \mathrm{C}_{i+1} \mathrm{C}_{i}-\mathrm{C}_{i}=\mathrm{C}_{i+1} \mathrm{C}_{i} \mathrm{C}_{i+1}-\mathrm{C}_{i+1} \text { for } 1 \leqslant i \leqslant n-2 .
\end{array}\right.
$$

For a permutation $y: i \mapsto y_{i}$ in $\mathfrak{S}_{n}$, let

$$
I=I_{y}=\left\{i \mid i<y_{i}\right\}, \quad J=J_{y}=\left\{j \mid j>y_{j}\right\} .
$$

Since $\sum_{i=1}^{n}\left(i-y_{i}\right)=0$, we have immediately

$$
\operatorname{deg}\left(A_{y}^{+}\right)=\sum_{i \in I}\left(y_{i}-i\right)=\sum_{j \in J}\left(j-y_{j}\right)=\operatorname{deg}\left(A_{y}^{-}\right) .
$$

We fix an order on $I=\left\{i_{1}, \cdots, i_{s}\right\}(s=\# I)$ such that $y_{i_{1}}>\cdots>y_{i_{s}}$ and an order on $J=\left\{j_{1}, \cdots, j_{t}\right\}(t=\# J)$ such that $j_{1}<\cdots<j_{t}$. For any $i \in I$ and $j \in J$ we put

$$
\mathrm{e}_{\left[i, y_{i}\right)}=\mathrm{e}_{i} \mathrm{e}_{i+1} \cdots \mathrm{e}_{y_{i}-1}, \quad \mathbf{f}_{\left(j, y_{j}\right]}=\mathbf{f}_{j-1} \mathbf{f}_{j-2} \cdots \mathbf{f}_{y_{j}} .
$$


Here for $a<b$ and $c>d$, we use the notation

$$
[a, b):=\{a, a+1, \cdots, b-1\}, \quad(c, d]:=\{c-1, c-2, \cdots, d\} .
$$

Then

$$
\mathrm{e}^{\left(A_{y}^{+}\right)}=\mathrm{e}_{\left[i_{1}, y_{i_{1}}\right)} \cdots \mathrm{e}_{\left[i_{s}, y_{i_{s}}\right)}, \quad \mathrm{f}^{\left(A_{y}^{-}\right)}=\mathrm{f}_{\left(j_{1}, y_{j_{1}}\right]} \cdots \mathrm{f}_{\left(j_{t}, y_{j_{t}}\right]} .
$$

Theorem 9.4. For any $w \in \mathfrak{S}_{n}$, there is a reduced expression $w=s_{i_{1}} \cdots s_{i_{l}}$ satisfying

$$
\mathrm{m}^{\left(A_{w}\right)}=\mathrm{C}_{i_{1}} \cdots \mathrm{C}_{i_{l}} .
$$

Proof. For $i=1, \cdots, n$, identify $\mathfrak{S}_{i}$ with the subgroup of $\mathfrak{S}_{n}$ generated by $s_{1}, \cdots$, $s_{i-1}$. We induct on the smallest integer $m$ such that $w \in \mathfrak{S}_{m}$. If $m=1$, then $w=1$; so $\mathrm{m}^{\left(A_{w}\right)}=\mathrm{k}_{\omega}$, the identity element in $H$, and the result is clear. Thus, assume, for some $m>1$, that $\mathrm{m}^{\left(A_{z}\right)}=\mathrm{C}_{i_{1}} \cdots \mathrm{C}_{i_{l}}$ whenever $z \in \mathfrak{S}_{m-1}$. For notational simplicity, we take $m=n$. It suffices to prove that if $z=w s_{n-1} \cdots s_{i}$ for some $i, i \leqslant n$ and some $w \in \mathfrak{S}_{n-1}$, then $\mathrm{m}^{\left(A_{z}\right)}=\mathrm{m}^{\left(A_{w}\right)} \mathrm{C}_{n-1} \cdots \mathrm{C}_{i} 10$ In the case $i=n$, this means just that $z=w \in \mathfrak{S}_{n-1}$, and the result is true by the inductive hypothesis. So we proceed by downward induction on $i$.

Write $A_{n}=A_{w}$, and for $1 \leqslant i \leqslant n-1$, set

$$
A_{i}=A_{i+1} A_{s_{i}}=A_{n} A_{s_{n-1}} \cdots A_{s_{i}} .
$$

Since $A_{i}$ is obtained from $A_{i+1}$ by switching the $i$ th and $(i+1)$ th columns of $A_{i}$, the permutation matrix $A_{i}$ has a 1 in the $(n, i)$-position. Also, the entries in the first $i-1$ columns of $A_{i}$ and $A_{w}$ agree identically. In addition, the $i$ th, $\cdots,(n-1)$ thcolumns of $A_{w}$ identify with the $(i+1)$ th, $\cdots, n$th columns of $A_{i}$. Simply put, the matrix $A_{i}$ is obtained from the matrix $A_{w}$ by cyclically permuting to the right the last $n-i+1$ columns of $A_{w}$.

Put $y=w s_{n-1} \cdots s_{i+1}$, so that $A_{i+1}=A_{y}$. Write $B=\left(b_{i, j}\right)=A_{y}$ for simplicity. We can assume that $\mathrm{m}^{\left(A_{y}\right)}=\mathrm{m}^{\left(A_{w}\right)} \mathrm{C}_{n-1} \cdots \mathrm{C}_{i+1}$. By the notation introduced before the statement of the theorem, $j_{t}=n(t=\# J)$ and $y_{j_{t}}=i+1$. We must consider two cases:

Case 1. Suppose $i \neq y_{a}$ for all $a \in J$. Thus, the 1 appearing in column $i$ of $B$ does not appear in $B^{-}$. It is either on the diagonal or above the diagonal. That is, there exists $k \leqslant i$ such that $b_{k, i}=1$.

Case 1a. If $i \notin\left(j_{a}, y_{j_{a}}\right]$ for all $a=1, \cdots, t$, then $\mathrm{e}_{i}$ commutes with every factor $f_{\left(j, y_{j}\right]}$ appearing in $\mathrm{f}^{\left(B^{-}\right)}$, and hence

$$
\mathrm{m}^{(B)} \mathrm{C}_{i}=\mathrm{k}_{\omega} \mathrm{e}^{\left(B^{+}\right)} \mathrm{f}^{\left(B^{-}\right)} \mathrm{e}_{i} \mathrm{f}_{i} \mathrm{k}_{\omega}=\mathrm{k}_{\omega} \mathrm{e}^{\left(B^{+}\right)} \mathrm{e}_{i} \mathrm{f}^{\left(B^{-}\right)} \mathrm{f}_{i} \mathrm{k}_{\omega} .
$$

Since the 1 in the $i$-th column of $B$ is not in $B^{-}$,

$$
\mathrm{f}^{\left(B^{-}\right)} \mathbf{f}_{i}=\mathrm{f}^{\left(A_{i}^{-}\right)} \text {. }
$$

If $k=i$, then $\left(A_{i}\right)_{i, i+1}=1\left(=\left(A_{i}\right)_{n, i}\right)$ and

$$
\mathrm{e}^{\left(B^{+}\right)} \mathrm{e}_{i}=\mathrm{e}_{\left[i_{1}, y_{i_{1}}\right)} \cdots \mathrm{e}_{\left[i_{a}, y_{i_{a}}\right)} \mathrm{e}_{i} \mathrm{e}_{\left[i_{a+1}, y_{i_{a+1}}\right)} \cdots \mathrm{e}_{\left[i_{s}, y_{i_{s}}\right)},
$$

where $y_{i_{a+1}}<i \leqslant y_{i_{a}}$ : if $i=y_{i_{a}}$, then the $i$-th column of $B$ would have two 1 's, which is impossible. Thus, $i<y_{i_{a}}$ and $\mathrm{e}^{\left(B^{+}\right)} \mathrm{e}_{i}=\mathrm{e}^{\left(A_{i}^{+}\right)}$. Now 9.4 .2 gives $\mathrm{m}^{(B)} \mathrm{C}_{i}=\mathrm{m}^{\left(A_{i}\right)}$.

\footnotetext{
${ }^{10}$ Here we use the fact that if $w=t_{1} \cdots t_{u}$ is a reduced expression for $w$, then $z=$ $t_{1} \cdots t_{u} s_{n-1} \cdots s_{i}$ is a reduced expression for $z$.
} 
If $k<i$, then $i=y_{a}$ for some $a \in I$ and

$$
\mathrm{e}^{\left(B^{+}\right)}=\mathrm{e}_{\left[i_{1}, y_{i_{1}}\right)} \cdots \mathrm{e}_{[a, i)} \cdots \mathrm{e}_{\left[i_{s}, y_{i_{s}}\right)} .
$$

Since $\mathrm{e}_{i}$ commutes with all the factors on the right-hand side of $\mathrm{e}_{[a, i)}$ and $\mathrm{e}_{[a, i)} \mathrm{e}_{i}=$ $\mathrm{e}_{[a, i+1)}$, it follows that $\mathrm{e}^{\left(B^{+}\right)} \mathrm{e}_{i}=\mathrm{e}^{\left(A_{i}^{+}\right)}$, and hence, combining 0.4.2 $\mathrm{m}^{(B)} \mathrm{C}_{i}=\mathrm{m}^{\left(A_{i}\right)}$.

Case 1b. If $i \in\left(j_{a}, y_{j_{a}}\right]$ for some $a \in J$ (perhaps more than one), then $j_{a}>i>$ $y_{j_{a}}$. If $b$ is the largest among those $a$ 's, the monomial $\mathrm{m}=\prod_{b<a<t} \mathrm{f}_{\left(j_{a}, y_{j_{a}}\right]}(t=\# J)$ does not involve $\mathbf{f}_{i}$. Hence, $\mathrm{m}$ does not involve $\boldsymbol{f}_{i-1}$ and $\mathbf{f}_{i+1}$ : if $\mathbf{f}_{i-1}$ is involved, then $j_{a}-1=i-1$ and so $j_{a}=i$ for some $a>b$, but $j_{b}>i$, which is absurd since $j_{b}<j_{a}$; if $\mathrm{f}_{i+1}$ is involved, then $i+1=j_{y_{a}}$ for some $b<a<t$ and so column $i+1$ of $B$ has two 1's. Thus, by 4.1 ,

$$
\begin{aligned}
\prod_{b \leqslant a \leqslant t} \mathbf{f}_{\left(j_{a}, y_{j_{a}}\right]} \mathbf{e}_{i} \mathbf{f}_{i} & =\mathbf{f}_{\left(j_{b}, y_{j_{b}}\right]} \mathbf{e}_{i} \prod_{b<a \leqslant t} \mathbf{f}_{\left(j_{a}, y_{j_{a}}\right]} \mathbf{f}_{i} \\
& =\mathbf{f}_{j_{b}-1} \cdots\left(\mathbf{f}_{i} \mathbf{e}_{i}\right) \mathbf{f}_{i-1} \cdots \prod_{b<a \leqslant t} \mathbf{f}_{\left(j_{a}, y_{j_{a}}\right]} \mathbf{f}_{i} .
\end{aligned}
$$

Since $j_{t}=n$ and $y_{j_{t}}=i+1$, it follows that $\partial_{i}\left(\mathbf{f}_{i-1} \cdots \prod_{b<a \leqslant t} \mathbf{f}_{\left(j_{a}, y_{j_{a}}\right]} \mathbf{f}_{i}\right)=0$. By $0.2(2), \prod_{a \geqslant b} f_{\left(j_{a}, y_{j}\right]} e_{i} f_{i} k_{\omega}=e_{i} \prod_{a \geqslant b} f_{\left(j_{a}, y_{j_{a}}\right]} \mathrm{f}_{i} \mathrm{k}_{\omega}$. A similar argument, using induction and repeatedly applying [9.2, implies that $e_{i}$ "commutes" with every $\mathrm{f}_{\left(j_{a}, y_{j_{a}}\right]}$ with $i \in\left(j_{a}, y_{j_{a}}\right]$ and $i<j_{a}-1$. Finally, if $i=j_{a}-1$ occurs, then for any $1 \leqslant b \leqslant a-1$ we have $i \notin\left(j_{b}, y_{j_{b}}\right]$. So, after switching $\mathrm{e}_{i}$ with $\mathrm{f}_{\left(j_{a}, y_{j_{a}}\right]}$, which is possible using $9.2(2)$ again, we may commute $e_{i}$ with the rest of the product. This proves 9.4.1 in this case. The rest of the argument is entirely similar to the previous case. Therefore, we eventually obtain $\mathrm{m}_{(B)} \mathrm{C}_{i}=\mathrm{m}^{\left(A_{i}\right)}$.

Case 2. Suppose $i=y_{j}$ for some $j \in J$. Then the 1 in column $i$ of $B$ appears in $B^{-}$. So there exists $k \in J, k>i$, with $b_{k, i}=1$. This implies that $B^{+}=A_{i}^{+}$. Let $k=j_{a}$. For any $a<b \leqslant t$, if $i \in\left(j_{b}, y_{j_{b}}\right]$, then $i \neq y_{j_{b}}$. We claim that $i<j_{b}-1$. Suppose the contrary: $i=j_{b}-1$. Then $j_{b}=i+1$ and so $k=j_{a}<j_{b}=i+1$, which is absurd since $k>i$. Thus, from the argument in Case $1 \mathrm{~b}$, we see that

$$
\left(\prod_{a<b \leqslant t} \mathrm{f}_{\left(j_{b}, y_{j_{b}}\right]}\right) \mathrm{e}_{i} \mathrm{f}_{i} \mathrm{k}_{\omega}=\mathrm{e}_{i}\left(\prod_{a<b \leqslant t} \mathrm{f}_{\left(j_{b}, y_{j_{b}}\right]}\right) \mathrm{f}_{i} \mathrm{k}_{\omega} .
$$

Therefore, we have

$$
\begin{aligned}
\mathrm{f}^{\left(B^{-}\right)} \mathrm{e}_{i} \mathrm{f}_{i} \mathrm{k}_{\omega} & =\mathrm{f}_{\left(j_{1}, y_{j_{1}}\right]} \cdots \mathrm{f}_{\left(j_{a}, i\right]} \mathrm{e}_{i} \cdots \mathrm{f}_{(n, i+1]} \mathrm{f}_{i} \mathrm{k}_{\omega} \\
& =\mathrm{f}_{\left(j_{1}, y_{j_{1}}\right]} \cdots \mathrm{f}_{\left(j_{a}, i+1\right]}\left(\mathrm{e}_{i} \mathrm{f}_{i}-\left[\begin{array}{c}
\tilde{\mathrm{k}}_{i} ; 0 \\
1
\end{array}\right]\right) \cdots \mathrm{f}_{(n, i+1]} \mathrm{f}_{i} \mathrm{k}_{\omega} \\
& =\mathrm{e}_{i} \mathrm{f}^{\left(B^{-}\right)} \mathbf{f}_{i} \mathrm{k}_{\omega}-\mathbf{f}_{\left(j_{1}, y_{j_{1}}\right]} \cdots \mathrm{f}_{\left(j_{a}, i+1\right]}\left[\begin{array}{c}
\tilde{\mathrm{k}}_{i} ; 0 \\
1
\end{array}\right] \cdots \mathrm{f}_{(n, i+1]} \mathrm{f}_{i} \mathrm{k}_{\omega} .
\end{aligned}
$$

Here the first term requires a commutation between $\mathbf{f}_{\left(j_{1}, y_{j_{1}}\right]} \cdots \mathbf{f}_{\left(j_{a}, i+1\right]}$ and $e_{i}$, which can be argued as above by 9.2

We claim that $\mathbf{f}^{\left(B^{-}\right)} \mathbf{f}_{i} \mathrm{k}_{\omega}=0$. Indeed, since $\mathrm{f}_{i} \mathrm{k}_{\omega}=\mathrm{k}_{\lambda\left(s_{i}\right)} \mathbf{f}_{i}$, it suffices to prove that $\mathrm{f}^{\left(B^{-}\right)} \mathrm{k}_{\lambda\left(s_{i}\right)}=0$. We observe that the $i$ th entry $\lambda_{i}^{(t)}$ of $\lambda^{(t)}:=\lambda\left(s_{i}\right)$ is 0 and, if $\mathrm{f}_{(n, i+1]} \mathrm{k}_{\lambda}=\mathrm{k}_{\lambda^{(t-1)}} \mathrm{f}_{(n, i+1]}$, then, by 4.8, the $i$ th entry $\lambda_{i}^{(t-1)}$ of $\lambda^{(t-1)}$ is also 0 . Inductively, for any $a<b<t$, if $\mathrm{f}_{\left(j_{b}, y_{j_{b}}\right]} \mathrm{k}_{\lambda^{(b)}}=0$, then we are done. Otherwise, we have $\mathbf{f}_{\left(j_{b}, y_{j_{b}}\right]} \mathbf{k}_{\lambda^{(b)}}=\mathbf{k}_{\lambda^{(b-1)}} \mathbf{f}_{\left(j_{b}, y_{j_{b}}\right]}$ such that $\lambda_{i}^{(b-1)}=0$. This is seen as follows: if 
$i \notin\left(j_{b}, y_{j_{b}}\right]$, then $i<y_{j_{b}}$ and $\lambda_{i}^{(b-1)}=\lambda_{i}^{(b)}=0$; if $i \in\left(j_{b}, y_{j_{b}}\right]$, then $\mathbf{f}_{i+1} \mathbf{f}_{i} \mathbf{f}_{i-1}$ is a factor of $\mathbf{f}_{\left(j_{b}, y_{j_{b}}\right]}$, which guarantees $\lambda_{i}^{(b-1)}=0$. In the worst case scenario, 4.12 (1) implies $\mathrm{f}_{(k, i]} \mathrm{k}_{\lambda^{(a)}}=0$, since $\lambda_{i}^{(a)}=0$.

On the other hand, the argument above shows that $\prod_{a<b<t} \mathbf{f}_{\left(j_{b}, y_{j_{b}}\right]}$ has the same number of $\mathrm{f}_{i-1}, \mathrm{f}_{i}$ and $\mathrm{f}_{i+1}$; while $\mathrm{f}_{(n, i+1]} \mathrm{f}_{i}$ has one $\mathrm{f}_{i+1}$ and one $\mathrm{f}_{i}$. By $9.2(1)$,

$$
\left[\begin{array}{c}
\tilde{\mathbf{k}}_{i} ; 0 \\
1
\end{array}\right] \prod_{a<b \leqslant t} \mathrm{f}_{\left(j_{b}, y_{j_{b}}\right]} \mathbf{f}_{i} \mathbf{k}_{\omega}=-\prod_{a<b \leqslant t} \mathbf{f}_{\left(j_{b}, y_{j_{b}}\right]} \mathbf{f}_{i} \mathrm{k}_{\omega} .
$$

Combining all these observations, we obtain

$$
\mathbf{f}^{\left(B^{-}\right)} \mathbf{e}_{i} \mathbf{f}_{i} \mathbf{k}_{\omega}=\mathbf{f}_{\left(j_{1}, y_{j_{1}}\right]} \cdots \mathrm{f}_{\left(j_{a}, y_{j_{a}}+1\right]} \cdots \mathbf{f}_{(n, i+1]} \mathbf{f}_{i} \mathbf{k}_{\omega}=\mathbf{f}^{\left(A_{i}^{-}\right)} \mathbf{k}_{\omega} .
$$

Since $B^{+}=A_{i}^{+}$, we have proved that $\mathrm{m}^{(B)} \mathrm{C}_{i}=\mathrm{m}^{\left(A_{i}\right)}$.

Corollary 9.5. The integral algebra $H$ is generated over $\mathcal{Z}$ by the $\mathrm{C}_{i}$, and hence, by the $\mathrm{T}_{i}$. Therefore, $H$ is isomorphic to the Hecke algebra $H\left(\mathfrak{S}_{n}\right)$ (1.0.1). In particular, the $\mathrm{C}_{i}$ together with the relations (9.3.2 form a presentation of $H$.

Proof. The first two assertions follow from 9.3 and 9.4. Thus, the generators and relations given in 9.3 form a presentation for $H$, easily giving the last assertion.

Remarks 9.6. (1) With this presentation, the monomial basis $\mathfrak{M}_{\omega}$ is a set of certain monomials in the $\mathrm{C}_{i}$. $C_{s_{i}}^{\prime}$

(2) In the notation of [15], $\mathrm{T}_{i}$ corresponds to $\tilde{T}_{s_{i}}=v^{-1} T_{s_{i}}$ and $\mathrm{C}_{i}$ corresponds to

\section{ACKNOWLEDGEMENT}

The authors thank the referee for providing the authors with a voluminous list of comments, and, in particular, for pointing out an incorrect statement in an earlier version of the paper.

\section{REFERENCES}

1. A. A. Beilinson, G. Lusztig and R. MacPherson, A geometric setting for the quantum deformation of $\mathrm{GL}_{n}$, Duke Math. J. 61 (1990), 655-677. MR 91m:17012

2. K. Bongartz, On degenerations and extensions of finite-dimensional modules, Adv. Math. 121 (1996), 245-287. MR 98e:16012

3. V. Chari and N. Xi, Monomial bases of quantized enveloping algebras, in "Recent developments in quantum affine algebras and related topics" (Raleigh, NC, 1998), 69-81, Contemp. Math., 248, Amer. Math. Soc., Providence, RI, 1999. MR 2001c:17023

4. B. Deng and J. Du, On bases of quantized enveloping algebras, to appear.

5. R. Dipper and G. James, The q-Schur algebra, Proc. London Math. Soc. 59 (1989), 23-50. MR 90g: 16026

6. R. Dipper and G. James, q-tensor space and $q$-Weyl modules, Trans. Amer. Math. Soc. 327 (1991), 251-282. MR 91m:20061

7. S. Doty and A. Giaquinto, Presenting quantum Schur algebras as quotients of the quantized universal enveloping algebras of $\mathfrak{g l}_{2}$, preprint.

8. S. Doty and A. Giaquinto, Presenting Schur algebras, International Mathematics Research Notices IMRN 2002:36 (2002) 1907-1944.

9. J. Du, A note on the quantized Weyl reciprocity at roots of unity, Alg. Colloq. 2 (1995), 363-372. MR 96m:17024

10. J. Du, q-Schur algebras, asymptotic forms, and quantum $S L_{n}$, J. Algebra 177 (1995), 385408. MR 96k:17021 
11. J. Du, B. Parshall and L. Scott, Quantum Weyl reciprocity and tilting modules, Comm. Math. Phys. 195 (1998), 321-352. MR 99k:17026

12. J. Du and B. Parshall, Linear quivers and the geometric setting for quantum $G L_{n}$, Indag. Math., in press.

13. J. Du and H. Rui, Based algebras and standard bases for quasi-hereditary algebras, Trans. Amer. Math. Soc. 350 (1998), 3207-3235. MR 99b:16027

14. R. Green, q-Schur algebras as quotients of quantized enveloping algebras, J. Algebra 185 (1996), 660-687. MR 97k:17016

15. D. Kazhdan and G. Lusztig, Representations of Coxeter groups and Hecke algebras, Invent. Math. 53 (1979), 165-184. MR 81j:20066

16. M. Jimbo, A q-analogue of $U(g l(N+1))$, Hecke algebras, and the Yang-Baxter equation, Lett. Math. Phy. 11 (1986), 247-252. MR 87k:17011

17. G. Lusztig, Modular representations and quantum groups, Contemp. Math. 82 (1989) 59-77. MR 90a:16008

18. G. Lusztig, Finite-dimensional Hopf algebras arising from quantized universal enveloping algebras, J. Amer. Math. Soc. 3 (1990), 257-296. MR 91e:17009

19. G. Lusztig, Canonical bases arising from quantized enveloping algebras, J. Amer. Math. Soc. 3 (1990), 447-498. MR 90m:17023

20. G. Lusztig, Aperiodicity in quantum affine $\mathfrak{g l}_{n}$, Asian J. Math. 3 (1999), 147-177. MR 2000i: 17027

21. G. Lusztig, Transfer maps for quantum affine $\mathfrak{s l}_{n}$, In Representations and quantizations (Shanghai, 1998), China High. Educ. Press, Beijing (2000), 341-356. MR 2002f: 17026

22. O. Schiffmann and E. Vasserot, Geometric construction of the global base of the quantum modified algebra of $\hat{\mathfrak{g l}}_{n}$, Transf. Groups 5 (2000), 351-360. MR 2001k:17029

23. M. Takeuchi, Some topics on $G L_{q}(n)$, J. Algebra 147 (1992), 379-410. MR 93b:17055

24. H. Wenzl, Hecke algebra of type $A_{n}$ and subfactors, Invent. Math. 92 (1988), 349-383. MR 90b: 46118

School of Mathematics, University of New South Wales, Sydney 2052, Australia

E-mail address: j.du@unsw.edu.au

URL: http://www.maths.unsw.edu.au/ jied

Department of Mathematics, University of Virginia, Charlottesville, Virginia 229044137

E-mail address: bjp8w@virginia.edu

$U R L:$ http://www.math.virginia.edu/ bjp8w 